\title{
Protein kinase LKB1 promotes RAB7-mediated neuropilin-1 degradation to inhibit angiogenesis
}

\author{
Imoh S. Okon, ${ }^{1}$ Kathleen A. Coughlan, ${ }^{1}$ Cheng Zhang, ${ }^{1}$ Cate Moriasi, ${ }^{1}$ Ye Ding, ${ }^{1}$ Ping Song, ${ }^{1}$ Wencheng Zhang, ${ }^{1}$ Guangpu Li, ${ }^{2}$ \\ and Ming-Hui Zou ${ }^{1,2}$ \\ 'Section of Molecular Medicine and 'Department of Biochemistry and Molecular Biology, College of Medicine, University of Oklahoma Health Sciences Center (OUHSC), Oklahoma City, Oklahoma, USA.
}

\begin{abstract}
After internalization, transmembrane receptors (TMRs) are typically recycled back to the cell surface or targeted for degradation. Efficient TMR trafficking is critical for regulation of several processes, including signal transduction pathways, development, and disease. Here, we determined that trafficking of the angiogenic receptor neuropilin-1 (NRP-1) is abrogated by the liver kinase B1 (LKB1), a serine-threonine kinase of the calcium calmodulin family. We found that aberrant NRP-1 expression in tumor cells from patients with lung adenocarcinoma is associated with decreased levels of LKB1. In cultured lung cells, LKB1 accentuated formation of a complex between NRP-1 and RAB7 in late endosomes. LKB1 specifically bound GTP-bound RAB7, but not a dominant-negative GDP-bound form of RAB7, promoting rapid transfer and lysosome degradation of NRP-1. siRNAmediated depletion of RAB7 disrupted the transfer of NRP-1 to the lysosome, resulting in recovery of the receptor as well as increased tumor growth and angiogenesis. Together, our findings indicate that LKB1 functions as a RAB7 effector and suppresses angiogenesis by promoting the cellular trafficking of NRP-1 from RAB7 vesicles to the lysosome for degradation. Furthermore, these data suggest that LKB1 and NRP-1 have potential as therapeutic targets for limiting tumorigenesis.
\end{abstract}

\section{Introduction}

LKB1 (also called STK11), a serine-threonine protein kinase of the calcium calmodulin family, is ubiquitously expressed in several tissues, including liver, heart, lung, and skeletal muscle. Among the diverse functions of LKB1 are the regulation of hematopoietic stem cell survival, mammalian target of rapamycin (mTOR), and AMP-activated protein kinase (AMPK) (1-4). As a stress-responsive gene (5), LKB1 displays varying effects under cell stress conditions, such as serum deprivation or hypoxia. The essential functions of LKB1 are best demonstrated by the fact that global $L k b 1$ knockout is embryonically lethal in mice (6). To date, more than 100 sporadic LKB1 mutations have been identified (mainly point mutations and large deletions), with $30 \%-34 \%$ of breast and lung cancer patients possessing LKB1 alterations (7-10). Peutz-Jeghers syndrome (PJS), an inherited autosomal disease characterized by gastrointestinal polyps, represents another classic example of the outcome of $L K B 1$ mutations (11). LKB1 has therefore emerged as a commonly altered gene in certain disease conditions and may represent a major and early event in cancer development. However, with respect to the specific roles of LKB1 in angiogenesis, reports are conflicting. Vascular abnormalities and enhanced VEGF expression in LKB1deficient mice and fibroblast cells have been observed (6). Others have reported LKB1-mediated attenuation of angiogenesis and related tumor processes in breast and lung tumors $(10,12)$. Conversely, proangiogenic LKB1 functions have also been observed $(13,14)$. These contradictory findings are due in part to cell types or animal models used, disease types or stages, methodology, and other experimental variations. Here, we explored the functions of

Conflict of interest: The authors have declared that no conflict of interest exists. Submitted: January 24, 2014; Accepted: July 25, 2014.

Reference information: / Clin Invest. 2014;124(10):4590-4602. doi:10.1172/JCI75371.
LKB1 to gain mechanistic insight and better understand its roles in tumor angiogenesis and growth.

The neuropilin family encompasses 2 distinct gene products, the transmembrane proteins neuropilin-1 (NRP-1) and NRP-2, with $44 \%$ sequence homology $(15,16)$. NRP1 is a well-described angiogenesis-associated gene, with additional roles in axon guidance, cell survival, migration, and invasion (17-22). NRP-1 serves as a receptor to both VEGF and semaphorin (SEMA3A) family members. Although NRP-1 displays coreceptor functions for tyrosine kinase receptors $(23,24)$, possession of a 40 -amino acid cytoplasmic domain confers independent NRP-1 signaling capability (25-27). Enhanced NRP-1 expression during tumorigenesis may represent an angiogenic switch, and the receptor remains an attractive therapeutic target. NRP-1 expression in endothelial and tumor cells has been associated with regulating VEGFR2 trafficking (28-31). However, the factors that regulate NRP-1 trafficking and the fate of the internalized receptor are less understood.

RAB GTPases play important roles in the regulation of membrane proteins, including transport vesicle formation, motility, and fusion (32-37). The expression of more than 60 different mammalian RAB family members $(31,35)$ highlights complex but highly specific functions. While certain RABs are ubiquitously expressed, others demonstrate tissue- or functionspecific expression (32). Canonical RAB7 membrane trafficking functions oscillate between active (GTP-bound) and inactive (GDP-bound) conformations. Active GTP-bound RAB7 binds to effectors in order to execute vesicle tethering, docking, and fusion $(33,34)$. Effectors possess important functions that are critical to the integrity of vesicle transport and cargo carriers. In this study, we report novel LKB1 effector function for active GTP-bound RAB7, promoting lysosome degradation of NRP-1 and attenuating tumor angiogenesis and growth. 


\section{Results}

LKB1 expression negatively correlates with NRP-1 levels in cancer and endothelial cells. Based on frequent LKB1 alterations in lung tumors (8), we assessed LKB1 expression in clinical lung adenocarcinoma specimens from males and females aged 48-72 years (Supplemental Table 1; supplemental material available online with this article; doi:10.1172/JCI75371DS1). We further assessed the expression of angiogenesis-associated genes in these tissues, namely, VEGF, VEGFR2, and NRP1. Immunohistochemistry (IHC) revealed stronger staining for LKB1 in normal versus tumor tissues (Figure 1A). In contrast, NRP-1 and VEGF showed an opposite expression pattern, with robust staining in tumor specimens compared with healthy tissues (Figure 1A). Interesting, VEGFR2 expression was weak in tumor and nontumor tissues, independent of the levels of either VEGF or NRP-1 (Figure 1A). In commercially obtained lung cancer lysates, immunoblot analysis showed that NRP-1 expression correlated with loss of LKB1 in $50 \%$ of the samples (data not shown). The expression profile of LKB1 and NRP-1 protein levels was next assessed in lung cancer cell lines obtained from patients spanning various ages, disease stages, and histologies (Supplemental Table 2). The normal "noncancer" MRC-9 lung cells strongly expressed LKB1, with weak NRP-1 levels (Figure 1B). However, A549 cells exhibited a deficiency of LKB1, in sharp contrast to their NRP-1 expression, whereas H1299 or H1703 cells showed LKB1 expression and NRP-1 abrogation, as determined by immunoblotting (Figure 1B). With the exception of modest expression in H1792 cells, VEGFR2 was not detected across the cell lines (Figure 1B), which may suggest a negligible contribution to tumor-related processes in these cells. Importantly, a 2-fold decrease in NRP-1 protein expression was observed at 24 hours after transfection of LKB1 in A549 cells (Figure 1, C and D). Decreased NRP-1 expression in the presence of LKB1 was further confirmed using confocal microscopy (Figure 1E and Supplemental Figure 1). Indirect immunofluorescence using NRP-1 antibody showed less punctate-like staining in the cytoplasm of cells transfected with LKB1 compared with LacZ control (Figure 1E). Conversely, due to the transient nature of the transfection, LKB1 protein levels peaked at 24 hours after transfection, but decreased sharply by 48 hours, which correlated with the recovery of NRP-1 protein levels (Figure $1 F$ ). Real-time PCR (RT-PCR) quantification of LKB1-transfected cells revealed a slight decrease in NRP1 mRNA expression compared with the LacZ condition; however, this reduction was not sufficient to account for the observed decrease in NRP-1 protein levels (Figure $1 \mathrm{G}$ and Supplemental Figure 2).

We next determined whether the inverse LKB1/NRP-1 expression patterns observed in cancer cells also occurred within endothelial cells, which represent an essential component of angiogenesis. Human aorta endothelial cells treated with LKB1 siRNA exhibited increased NRP-1 expression levels compared with control siRNA treatment (Figure 1, H and I). Moreover, in vivo IHC staining of mouse aorta with a NRP-1 antibody demonstrated increased NRP-1 expression in mice with endothelialspecific LKB1 knockout compared with age-matched controls (Figure 1J and ref. 38). Together, these data support the negative correlation between LKB1 and NRP-1 expression in cancer and endothelial cells.
LKB1-mediated attenuation of NRP-1 is independent of VEGF. Due to the hypoxic microenvironment of solid tumors, and the concomitant upregulation of VEGF expression (39), we tested the response of NRP-1 to these factors. In contrast to transfection with empty vector control (LacZ), A549 cells transfected with LKB1 demonstrated abrogation of NRP-1 protein expression in the presence of exogenous VEGF stimulation at 5 or 30 minutes (Figure 2, A and B). Consistent with a previous report (40), we confirmed decreased NRP-1 expression under hypoxia treatment compared with normoxia (Supplemental Figure 3). Importantly, in the presence of LKB1 expression, NRP-1 protein levels were further reduced (Figure 2, A and B, and Supplemental Figure 4), suggesting a role for LKB1 in mediating NRP-1 protein degradation.

Next, we assessed RNA and protein expression of VEGF in LacZ- or LKB1-transfected cells with or without hypoxia. RTPCR quantification demonstrated attenuated VEGF expression by LKB1 transfection (Figure 2C). Consistent with previous observations (6), we confirmed decreased VEGF protein expression in LKB1-transfected cells (Supplemental Figure 5). Furthermore, secreted VEGF was reduced in LKB1- versus LacZ-transfected cells under normoxic and hypoxic conditions (Figure 2D). To test whether decreased NRP-1 expression in the presence of LKB1 was dependent on cellular VEGF (which is a major NRP-1 growth factor), we silenced expression of VEGF in A549 cells by siRNA. While NRP-1 expression remained unaffected in LKB1-null cells, decreased levels of the receptor were evident in LKB1-positive cells, independent of VEGF (Figure 2, E and F). Negligible expression of VEGF was evident in this cell line, in contrast to that of NRP-1 (Figure 2E). Weak expression of VEGF compared with robust endogenous NRP-1 expression was further confirmed in a subset of lung cancer cell lines (Figure 2, G and H), supporting independent NRP-1 tumorpromoting processes in these cell lines. Importantly, these data indicate that negative regulation of NRP-1 expression instigated by LKB1 is independent of VEGF.

LKB1 promotes degradation of NRP-1 via the lysosome machinery. To delineate the mechanism(s) that contribute to dramatic loss of NRP-1 expression in the presence of LKB1, we investigated NRP-1 degradation in H1792 cells, which possess endogenous LKB1 expression. Cells were treated with the proteasome inhibitor $\mathrm{MG}-132$ or the lysosome inhibitor chloroquine for 3 hours, which demonstrated 2-fold increased NRP-1 protein expression with addition of the lysosome inhibitor compared with untreated cells (Figure 3, A and B). In the presence of hypoxia, 2 potent but structurally unrelated proteasome inhibitors were tested (MG-132 and lactacystin), but failed to rescue NRP-1 protein expression, as assessed by immunoblotting (Figure 3C and Supplemental Figure 6). However, lysosome inhibition with chloroquine or bafilomycinA (BafA1) resulted in NRP-1 accumulation (Figure 3D and Supplemental Figure 7), which suggested that the lysosome machinery may be critical for NRP-1 degradation. Rescued NRP-1 expression upon lysosome inhibition was confirmed in LKB1-transfected A549 cells. First, immunofluorescence using anti-NRP-1 antibody demonstrated loss of punctate NRP-1 staining in LKB1-transfected cells, consistent with our previous data (Figure 1E); however, upon addition of BafA1, NRP-1 recovery 
A

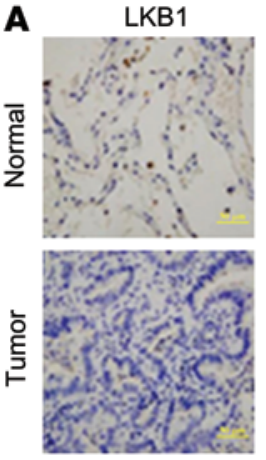

C

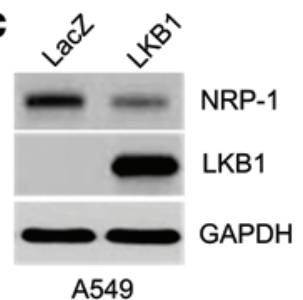

$\mathbf{F}$
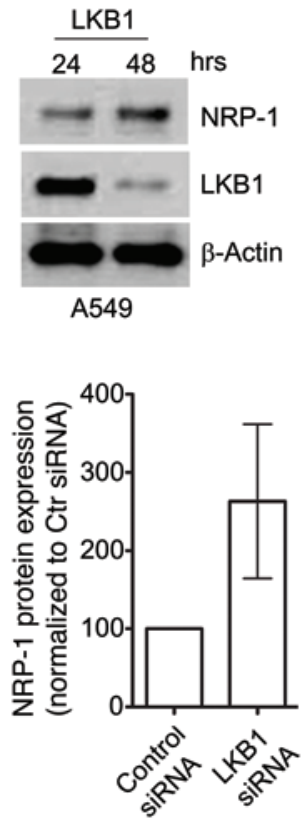

VEGF
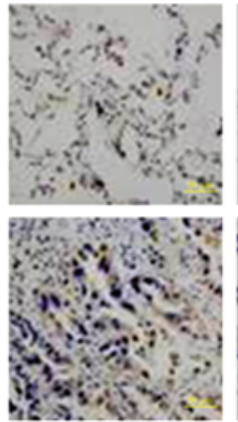

VEGFR2

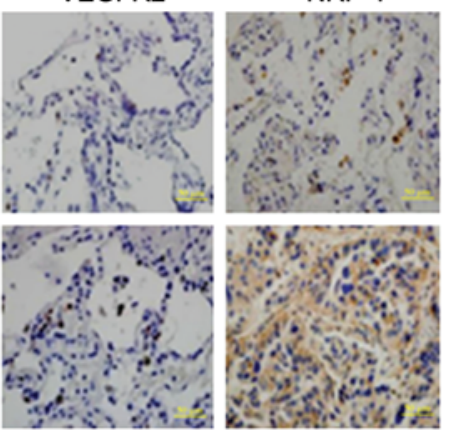

B

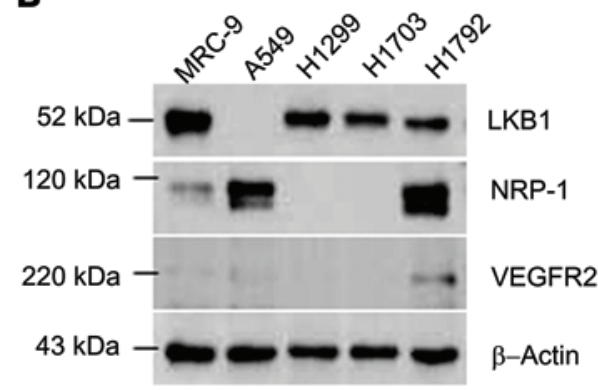

E
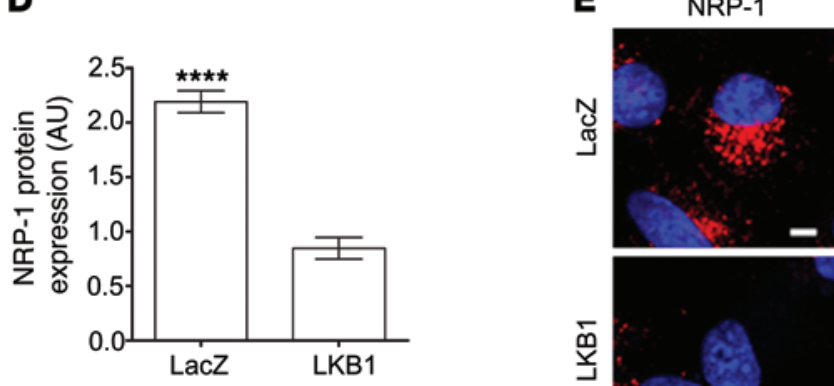

MERGE

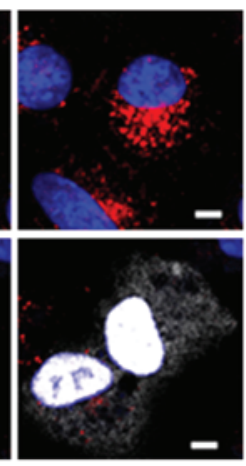

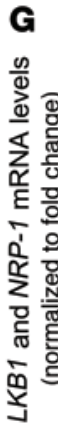
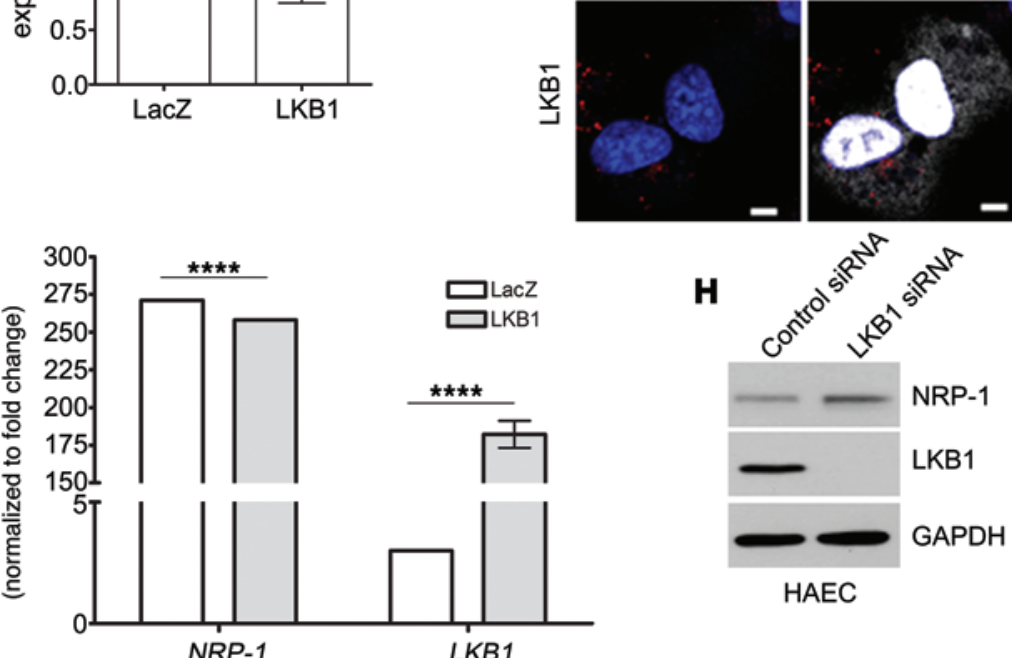

J

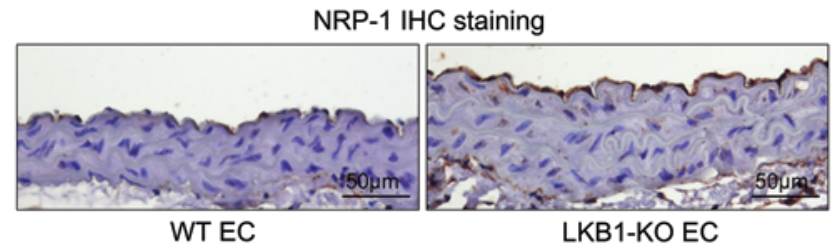

Figure 1. Negative correlation between LKB1 and NRP-1 expression. (A) Paraffin-embedded matched normal (control) and lung adenocarcinoma specimens were stained using primary antibodies for LKB1, NRP-1, VEGF, and VEGFR2 by IHC. Tissues were stained using secondary antibody HRPDAB detection reagents (brown) and counterstained with hematoxylin (blue). Scale bars: $50 \mu \mathrm{m}$. (B) Immunoblot analysis detecting LKB1, NRP-1, and VEGFR2 protein levels in A549, H1299, H1703, and H1792 lung cancer cell lines and "normal" noncancer MRC-9 cells. (C and D) Immunoblot analysis and detection of NRP-1 levels in A549 cell lysate after transient transfection (24 hours) with LacZ or LKB1 expression plasmids under basal, serumstarved conditions. Mean \pm SD is shown $(n=5)$. (E) NRP-1 expression (red) was visualized by indirect immunofluorescence after transient transfection (24 hours) with LacZ or LKB1 (white). DAPI (blue) represents nuclear staining. Scale bars: $5 \mu \mathrm{m}$. (F) Immunoblot analysis and detection of NRP-1 levels in A549 cell lysate after transient transfection with LKB1 or LacZ expression plasmids for 24 or 48 hours. (C) NRP1 and LKB1 mRNA expression, detected by RT-PCR, in LKB1- or LacZ-transfected A549 cells; mean \pm SD is shown $(n=3)$. (H and I) LKB1 expression was silenced (siRNA) in human aortic endothelial cells (HAEC), and NRP-1 protein level was detected by immunoblotting. Mean \pm SD is shown $(n=3)$. (J) IHC staining for NRP-1 in the aorta derived from endothelial-specific LKB1 knockout mice. Tissues were stained using secondary antibody HRP-DAB detection reagents (brown) and counterstained with hematoxylin (blue). Scale bars: $50 \mu \mathrm{m} .{ }^{* * *} P<0.0001$. 
A

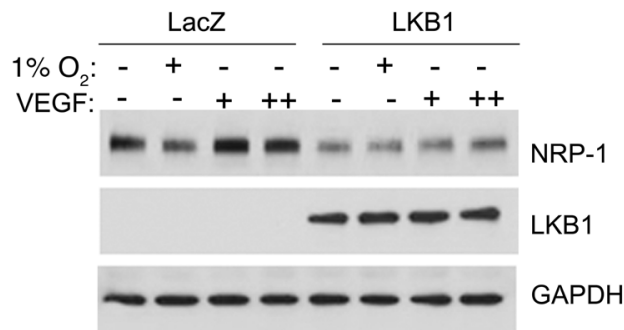

C

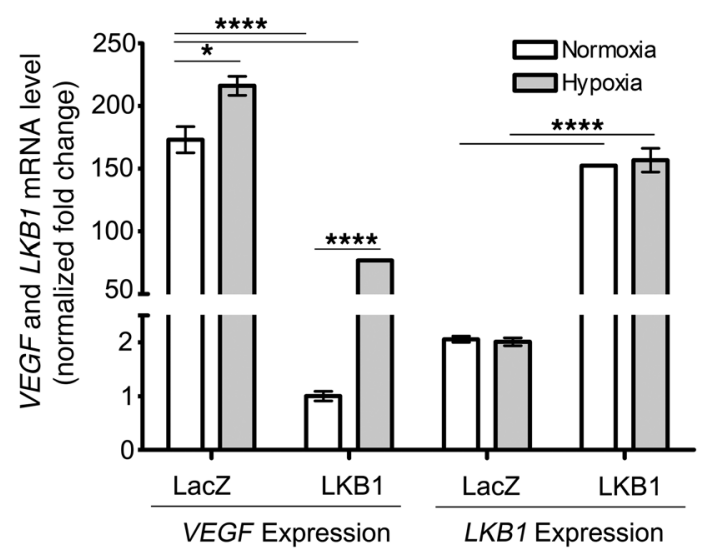

B

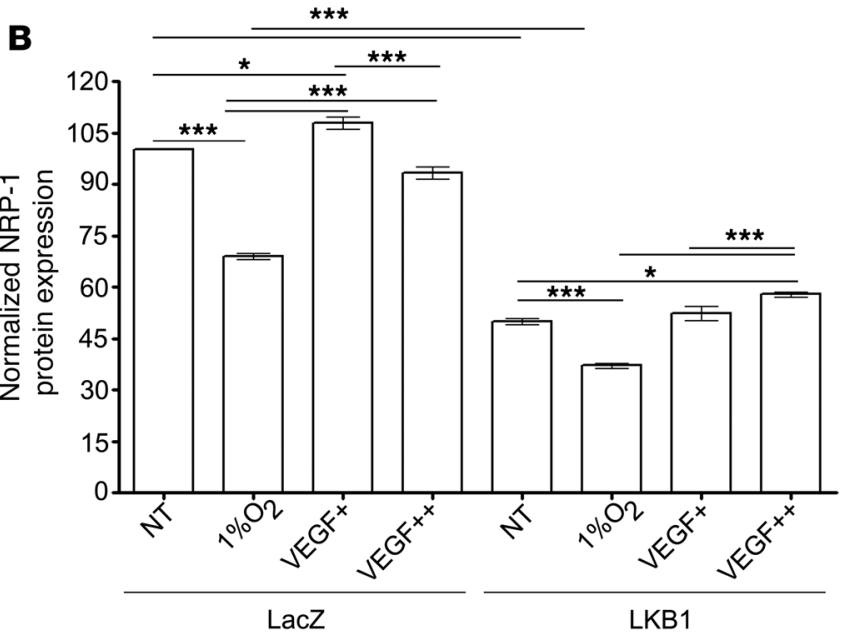

D

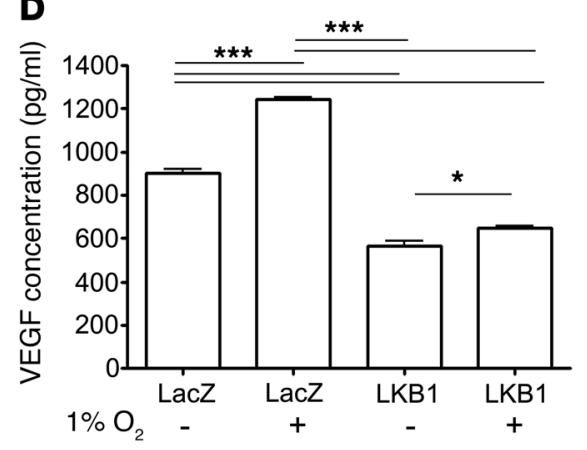

E

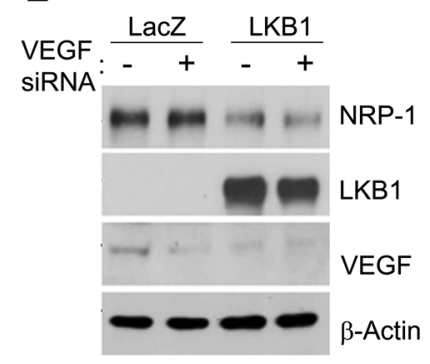

$\mathbf{F}$

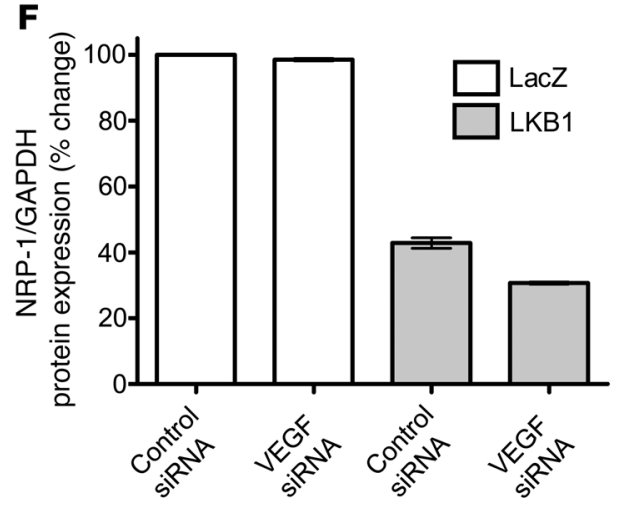

G

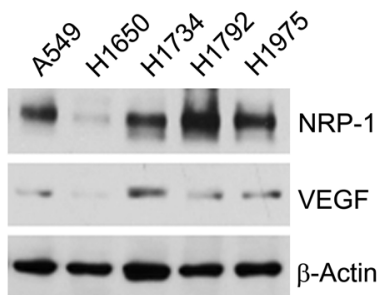

H

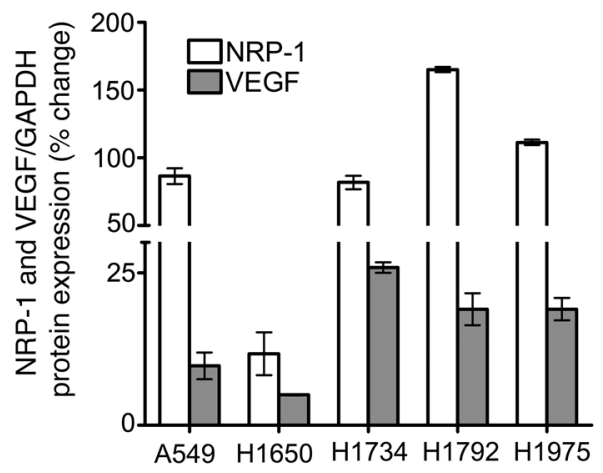

Figure 2. LKB1 attenuates NRP-1 expression in a VEGF-independent manner. ( $\mathbf{A}$ and $\mathbf{B}$ ) Response of NRP-1 to hypoxia treatment (1\% $\mathrm{O}_{2} ; 3$ hours) or VEGF stimulation (25 ng/ml for 5 [+] or 30 [++] minutes) in LacZ- or LKB1-transfected A549 cells was assessed by immunoblot. Mean \pm SD is shown $(n=3)$. (C) Under normal or hypoxia treatment (3 hours), NRP1 and LKB1 mRNA expression was assessed by RT-PCR in LacZ- or LKB1-transfected A549 cells. Mean \pm SD is shown $(n=3)$. (D) Under the same experimental conditions, secreted VEGF levels were measured by ELISA; mean \pm SD is shown $(n=4)$. ( $E$ and $\mathbf{F})$ VEGF expression was silenced for 48 hours (siRNA) in LacZ- or LKB1-transfected A549 cells, and NRP-1 levels were assessed by Western blot. Mean \pm SD is shown $(n=2)$. ( $\mathbf{G}$ and $\mathbf{H})$ Expression of VEGF and NRP-1 in various lung cancer cell lines was measured by immunoblot; mean \pm SD is shown $(n=2) .{ }^{*} P<0.05,{ }^{* *} P<0.001,{ }^{* * * *} P<0.0001$.

was visualized as punctate-like vesicle structures within the cytoplasm (Figure 3E). Furthermore, in LKB1-transfected A549 cells, we observed significant recovery of NRP-1 protein expression in cells treated with BafA1 compared with vehicle control (Figure 3, F and G). Together, these data support a role for LKB1 in mediating NRP-1 degradation via the lysosome.
Nucleocytoplasmic LKB1 transport and activity is required for attenuation of NRP-1 expression. Due to its predominant localization in the nucleus, the transport and cytosolic distribution of LKB1 is required for activation, where it complexes with STRAD and MO25 (41-44). We previously demonstrated that phosphorylation of LKB1 is required for its nucleocytoplasmic transport $(41,42)$. 
In H1792 cells, we observed LKB1 phosphorylation at the canonical serine 428 residue under hypoxic conditions (data not shown). We examined the spatial localization of LKB1 under normoxic and hypoxic conditions using cell fractionation experiments in A549 cells. Enhanced cytosolic LKB1 distribution in response to hypoxia compared with normoxia was verified by confocal microscopy, which demonstrated predominant perinuclear LKB1 staining (Figure 4, A and B, and Supplemental Figure 8). To further understand the importance of LKB1 distribution on NRP-1 degradation, we used the constitutively nuclear-localized LKB1 mutant SL-26, which was originally identified in PJS patients (42). In transfected A549 cells, immunofluorescence verified the spatial distribution of SL-26 as predominately nuclear localized, compared with wild-type LKB1 (nuclear and cytoplasmic), under normoxic conditions (Figure 4C). Next, we assessed the cellular distribution of SL-26 under hypoxic conditions using cell fractionation and confocal microscopy. We observed minimal SL-26 expression in the cytoplasmic fraction upon hypoxia treatment, which correlated with NRP-1 expression (Figure 4, D and E, and Supplemental Figure 9).

Next, we determined whether the SL-26 (constitutively nuclear-localized), S428A (point mutation at phosphorylation site 428), and D194A (kinase-inactive) LKB1 mutants can interact with NRP-1, leading to its degradation through the lysosomal pathway. After immunoprecipitation using the LKB1 mutants, weak interaction was detected, compared with the strong NRP-1 interaction with wild-type LKB1 (Figure 4F). Importantly, the abrogation of NRP-1 expression observed in cells transfected with wild-type LKB1 failed to occur in those transfected with the mutant constructs (Figure 4F). Confirmation of the NRP-1/LKB1 interaction was verified by performing a reverse LKB1 pulldown, followed by immunoblotting for NRP-1 (Figure $4 \mathrm{G}$ ). With the exception of the kinase-dead D194A mutant, LKB1 activity is not affected by LKB1 phosphorylation residue mutations (42). Therefore, our data suggest that, in addition to LKB1 activity, enhanced cytosolic distribution of LKB1 is required for the attenuation of NRP-1 expression.

Internalized NRP-1 localizes within RAB7 endosomes. Given the critical regulatory functions of RAB GTPases in the trafficking of membrane receptors (32-37), we assessed potential associations of NRP-1 with canonical early (RAB5), late (RAB7), or recycling (RAB11) endosomes. After serum deprivation, immunocytochemistry revealed partial punctate-like localization of NRP-1 with RAB7, but to a lesser extent than that with RAB5 or RAB11 (Figure 5A). Colocalization coefficients for NRP-1 with RAB5 (0.067; 6.7\%), RAB7 (0.21; 21.0\%), or RAB11 (0.03; 3.0\%) vesicles confirmed predominant and specific colocalization of NRP-1 with RAB7 (Figure 5C), which suggests that internalized NRP-1 localizes to multivesicular bodies and/or late endosomes. We also tested whether hypoxia or VEGF treatment affects the distribution of NRP-1 within the endosomal compartments. We found the overall distribution of NRP-1 in A549 cells upon VEGF stimulation was not significantly changed compared with untreated controls: the calculated colocalization coefficient of NRP-1 with RAB7 was 0.19 (19\%) under untreated conditions, compared with 0.17 (17\%) with 30 minutes of VEGF treatment (Supplemental Figure 10). Under hypoxia, increased NRP-1 colocalization with RAB7 was confirmed (Supplemental Figure 10), demonstrating that hypoxia increases NRP-1 internalization into the endosomal system, in agreement with a previous report (40). To further exclude NRP-1 localization in the early endosomes or recycling pathway, we investigated the colocalization of a well-established ligand, transferrin, which upon receptor binding is internalized into early endosomes, followed by trafficking through the RAB11 or RAB4 recycling pathway. NRP-1 showed little colocalization with transferrin (Supplemental Figure 11), which suggests that NRP-1 resides very little in the early endosomal pathway (5-minute time point) or the recycling pathway (30-minute time point).

The neuropilin family consists of not only NRP-1, but also NRP-2, which also plays an important role in cancer biology. Hence, we assessed whether NRP-2 colocalizes in RAB7-positive vesicles, similar to NRP-1. Colocalization coefficients demonstrated very little colocalization of NRP-2 with RAB5 (0.070; 7.0\%), RAB7 $(0.046 ; 4.6 \%)$, or RAB11 (0.059; 5.9\%) in A549 cells (Figure 5, B and C). Furthermore, Western blot analysis demonstrated that LKB1-mediated attenuation of NRP-1 was specific and did not affect NRP-2 expression levels (Figure 5, D and E). To further substantiate the differences in spatial distribution of the neuropilins, we found that colocalization between NRP-1 and NRP-2 isoforms was negligible (about 5\%; Figure 5, F and G), which supports the notion of differential modulation of the receptors by LKB1, in part through spatial distribution in RAB7-positive vesicles.

$L K B 1$ promotes interaction of $R A B 7$ with $N R P-1$. Subsequent siRNA-mediated depletion of RAB5, RAB7, or RAB11 expression significantly rescued NRP-1 levels specifically in RAB7-deficient cells, not in RAB5- or RAB11-depleted cells (Figure 6, A and B), verifying the role of RAB7 in LKB1-mediated NRP-1 degradation. The rescue of NRP-1 expression in LKB1-positive cells was evident upon RAB7 depletion, but not in the control siRNA group (Figure $6, \mathrm{C}-\mathrm{F}$ ). As expected, rescued NRP-1 expression was demonstrated upon lysosome inhibition in control cells (Figure 6, E and F). Rescued NRP-1 expression was not observed in RAB5- or RAB11depleted cells (data not shown), further supporting RAB7-specific involvement in trafficking of the receptor.

We next examined the interaction of RAB7 and NRP-1 in the presence or absence of LKB1 expression. Immunoprecipitation of RAB7 showed weak but specific binding to NRP-1 in LacZtransfected A549 cells (Figure 6G). However, the interaction between NRP-1 and RAB7 was amplified in LKB1-expressing cells (Figure 6G), suggestive of enhanced NRP-1/RAB7 association in the presence of LKB1. Reverse immunoprecipitation of NRP-1 confirmed specific and enhanced interaction with RAB7 in LKB1-positive cells (Figure 6H). Next, we transfected the SL-26 or D194A LKB1 mutants into A549 cells and assessed RAB7 and NRP-1 interaction. Interestingly, interaction of RAB7 with NRP-1 was observed in cells transfected with D194A, but was lacking in those transfected with SL-26 (Figure 6I). Furthermore, consistent with Figure 4H, both D194A and SL-26 failed to block NRP-1 expression compared with wild-type LKB1 (Figure 6I). In agreement with the Western blot data, we found that NRP-1 colocalized with RAB7 vesicles in D194A-transfected cells (Supplemental Figure 12), which indicates that the kinase-inactive LKB1 may not affect RAB7/NRP-1 interaction, but it is unable to shuttle NRP-1 to the lysosome for degradation. 
A

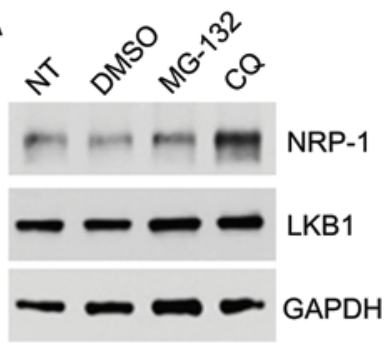

H1792

D

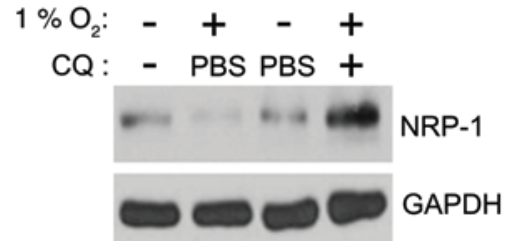

F

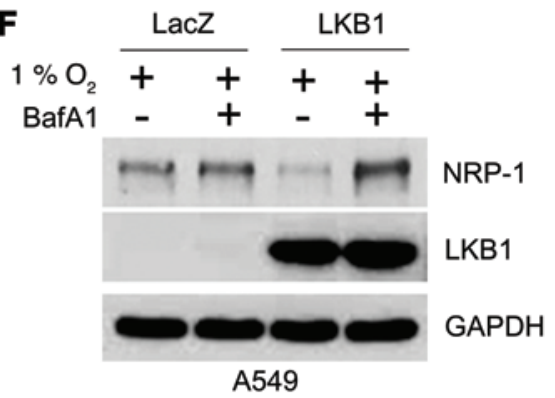

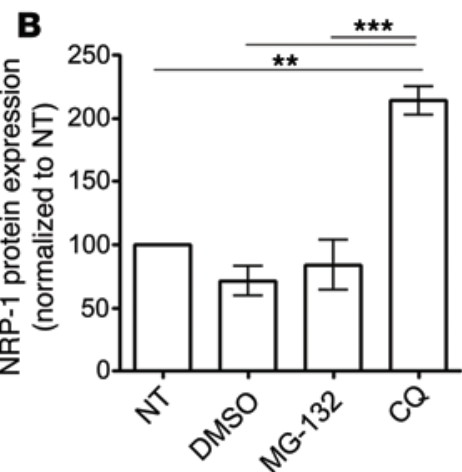

C

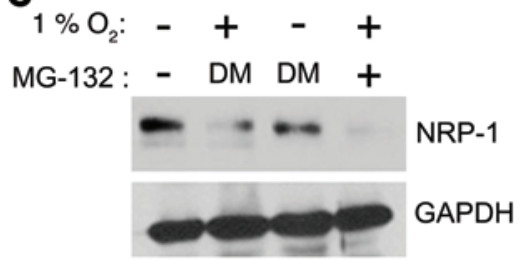

E

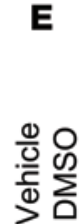

LKB1

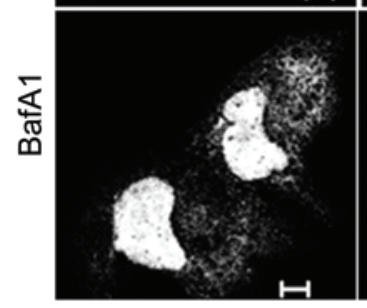

NRP-1

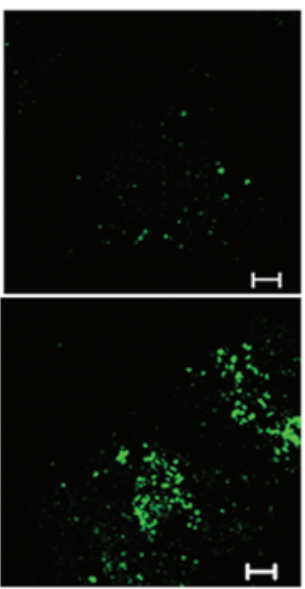

MERGE

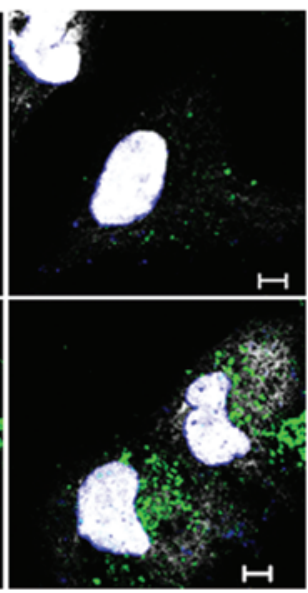

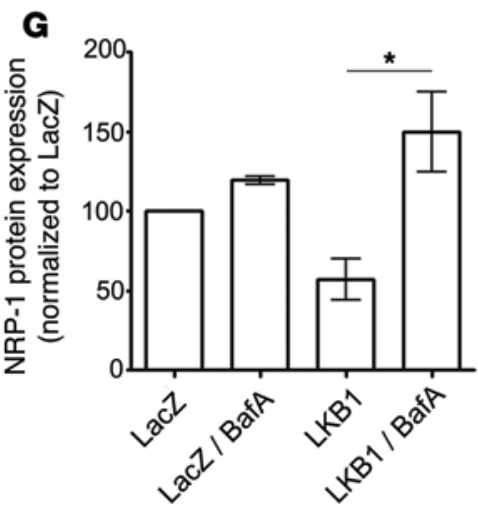

Figure 3. LKB1 promotes lysosome degradation of NRP-1. (A and B) Inhibition of major degradation pathways using $1.0 \mu \mathrm{M}$ proteasome (MG-132) or $100 \mu \mathrm{M}$ lysosome (chloroquine; CQ) inhibitors, and subsequent detection of NRP-1 levels by immunoblot analyses in $\mathrm{H} 1792$ cells. NT, not treated. Mean \pm SD is shown $(n=3)$. (C and $\mathbf{D})$ Proteasome or lysosome was inhibited (using MG-132 or chloroquine, respectiveIy) under normoxia vs. hypoxia, and NRP-1 expression was detected by Western blot. DM, DMSO.

(E) After lysosome inhibition with $0.1 \mu \mathrm{M}$ BafA1, NRP-1 expression (green) was visualized through indirect immunofluorescence after transient transfection (24 hours) of LKB1 (white) in A549 cells. DAPI (blue) represents nuclear staining. Scale bars: $5 \mu \mathrm{m}$. (F and $\mathbf{G})$. Following hypoxia treatment ( 3 hours), Western blot analyses was used to measure NRP-1 levels in the presence or absence of lysosome inhibition. Mean \pm SD is shown $(n=3) .{ }^{*} P<0.05,{ }^{* *} P<0.01$, ${ }^{* * *} P<0.001$.
Interaction of GTP-bound RAB7 with LKB1 is required for NRP1 abrogation. Next, we assessed the specific affinity of LKB1 for dominant-negative GDP-bound RAB7 (T22N construct) or for GTP-bound RAB7 (Q67L construct). Wild-type RAB7, in parallel with T22N and Q67L, was coexpressed with LKB1 in A549 cells. LKB1 preferentially interacted with Q67L, while demonstrating weak interaction with T22N (Figure 6J). Attenuated NRP-1 expression corresponded with the LKB1/Q67L cells, rather than the LKB1/T22N cells (Figure 6J). To confirm the importance of cytosolic LKB1 distribution, as well as to control for nonphysiological interactions in an overexpressed system, we demonstrated that SL-26 failed to interact with RAB7 constructs (Figure 6K). This result is consistent with the absence of cytosolic LKB1 distribution. Furthermore, NRP-1 expression remained unaffected in SL-26-expressing cell lysates (Figure 6K). Taken together, these results indicate that LKB1 nuclear export and subsequent complex formation with GTP-bound RAB7/NRP-1 result in enhanced lysosome degradation of NRP-1 (Figure 6L).

LKB1-mediated NRP-1 degradation results in decreased tumor growth and angiogenesis. Finally, we used in vivo mouse and chick animal models to investigate direct consequences of NRP-1 abrogation by LKB1. LKB1 and/or NRP-1 expression was stably silenced in $\mathrm{H} 1792$ lung cancer cells, and NRP-1 upregulation was found to be consistent with loss of LKB1 expression (Figure 7A). In subsequent in vivo experiments, cells with intact or deleted LKB1 and/or NRP-1 expression (LKB1 ${ }^{+} \mathrm{NRP}-1^{+}$, LKB1 $1^{+} \mathrm{NRP}-1^{-}$, LKB1-NRP-1 ${ }^{+}$, or LKB1-NRP-1') were implanted into chorioallantoic membrane (CAM) of fertilized chick embryos. Increased vessel density into the site of LKB1-NRP- $1^{+}$cell implants was apparent $(17.20 \pm 2.15$ vessels), in contrast to that in chicks receiving LKB1 ${ }^{+} \mathrm{NRP}-1^{-}, \mathrm{LKB}^{+}{ }^{+} \mathrm{NRP}-1^{+}$, and LKB1 ${ }^{-} \mathrm{NRP}-1^{-}$cells $(9.22 \pm 1.74$, $10.60 \pm 1.57$, and $8.60 \pm 1.7$ vessels, respectively; Figure 7, B and C). 
A

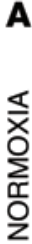

C
$\overline{\mathrm{m}}$

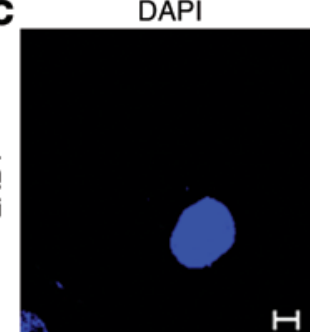

LKB1

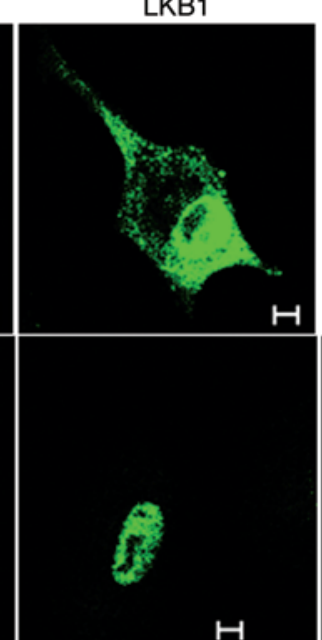

$\mathbf{F}$

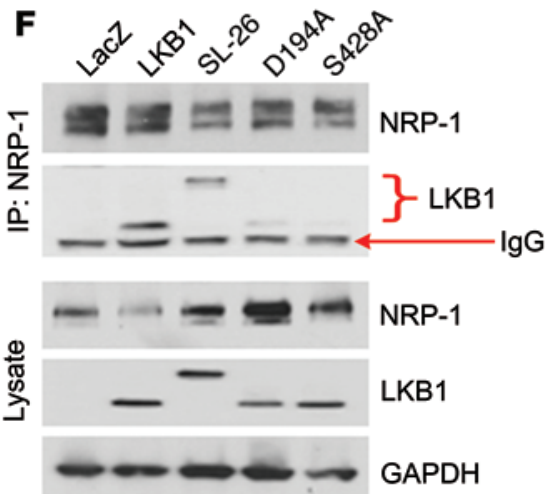

MERGE

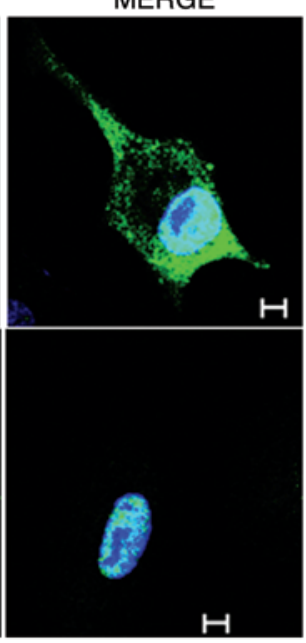

G

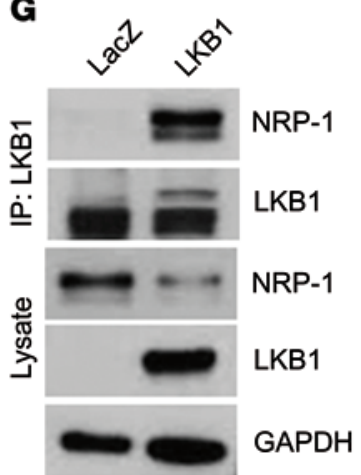

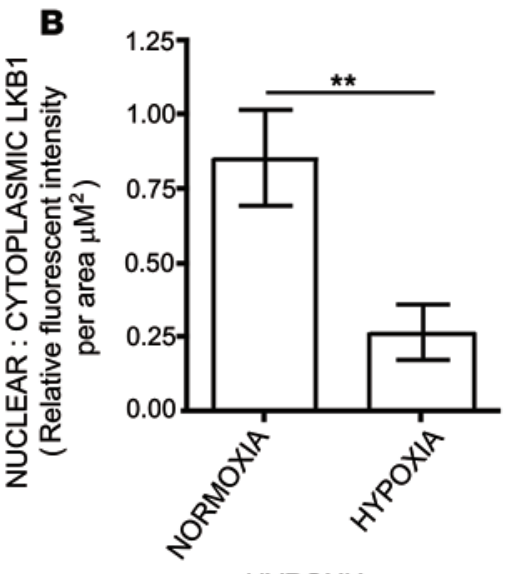

D
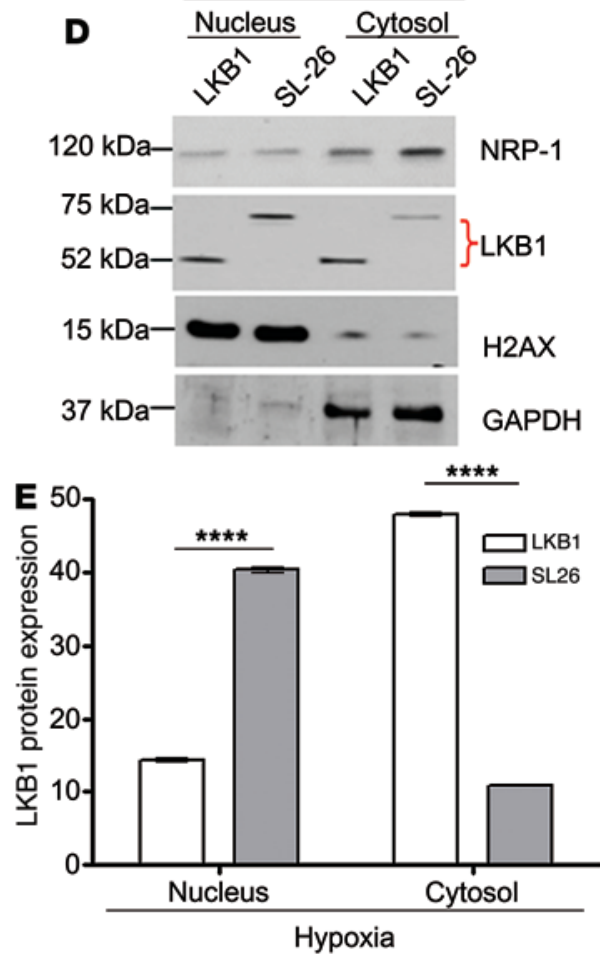

Figure 4. Hypoxia promotes LKB1 cytoplasmic distribution. (A) Immunofluorescence analysis of LKB1 (red) and DAPI (blue) under normoxic or hypoxic conditions in H1792 cells. Scale bars: $5 \mu \mathrm{m}$. (B) The nuclear/cytoplasmic ratio of LKB1 immunofluorescence in $\mathbf{A}$ was quantified; mean \pm SD is shown ( $n=10$ ). (C) Immunofluorescence analysis of wild-type LKB1 (green), the constitutively nuclear-localized SL-26 mutant (green), and DAPI (blue) under normoxia in transfected A549 cells. Scale bars: $5 \mu \mathrm{m}$. (D and E) Cytosolic and nuclear fractions of A549 cells transiently transfected with wild-type LKB1 or SL-26 were blotted for NRP-1, LKB1, and markers for different cell fractions. SL-26 possesses an EGFP tag. Mean \pm SD is shown $(n=3)$. (F) NRP-1 pulldown in A549 cells using the LKB1 mutants SL-26, D194A (kinase inactive), and S428A (point mutation at phosphorylation site 428). Interaction of NRP-1 and LKB1 were assessed in immunoprecipitated samples. (C) Reverse LKB1 pulldown and immunoblotting with NRP-1 and LKB1 antibodies. ${ }^{* *} P<0.01,{ }^{* * *} P<0.001$.

Tumor development after subcutaneous injection into nude mice showed that tumor growth occurred in $100 \%$ of mice injected with $\mathrm{LKB1}^{-} \mathrm{NRP}-1^{+}$and $\mathrm{LKB} 1^{-} \mathrm{NRP}-1^{-}$cells, in contrast to $40 \%$ of $\mathrm{LKB} 1^{+} \mathrm{NRP}-1^{-}$cell recipients (Figure $7, \mathrm{D}$ and $\mathrm{E}$ ). The tumors were significantly larger in mice injected with $\mathrm{LKB} 1^{-} \mathrm{NRP}-1^{+}$cells
$(30.40 \pm 5.46 \mathrm{mg})$ than mice with $\mathrm{LKB}^{+}{ }^{+} \mathrm{NRP}-1^{-}$cells $(4.86 \pm 3.08$ mg; Figure 7, D and F). Furthermore, only $20 \%$ of mice injected with $\mathrm{LKB} 1^{+} \mathrm{NRP}-1^{+}$cells developed tumors, and tumor size was smaller in these mice $(3.08 \pm 3.08 \mathrm{mg})$ than in those injected with $\mathrm{LKB1}^{+} \mathrm{NRP}-1^{-}$or LKB1-NRP-1 ${ }^{+}$cells (Figure 7, D and E). Although 


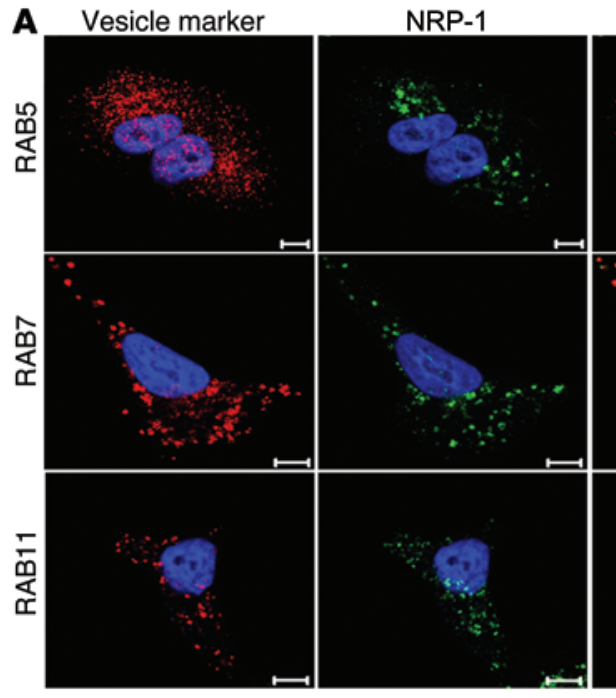

C

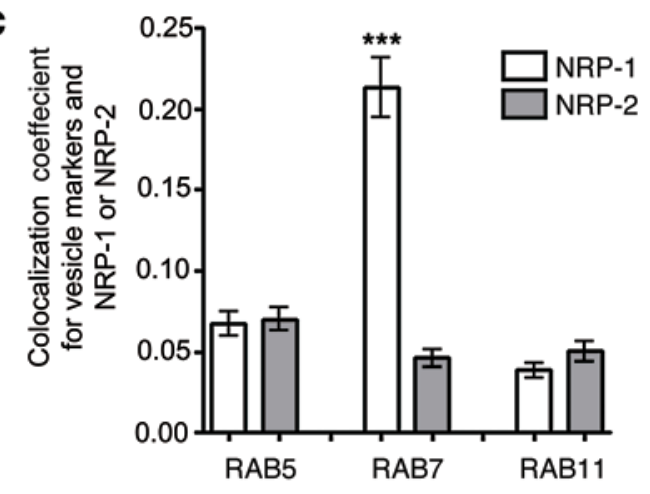

$\mathbf{F}$
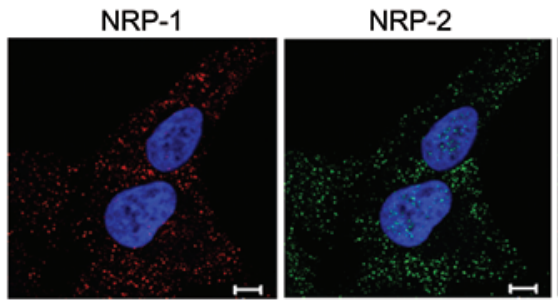

MERGE

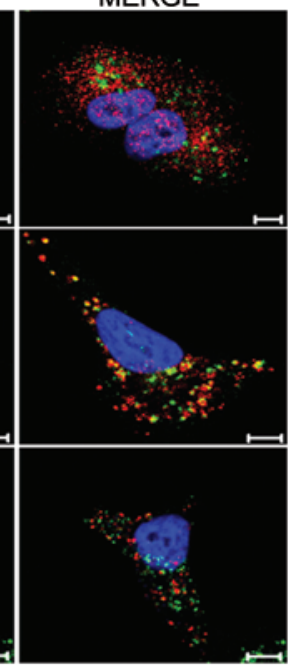

D
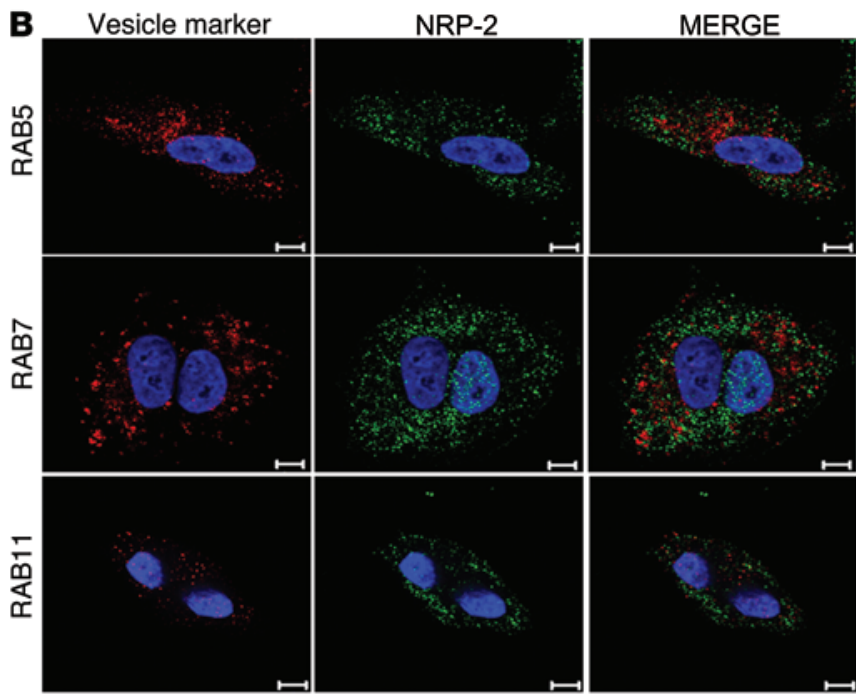

E
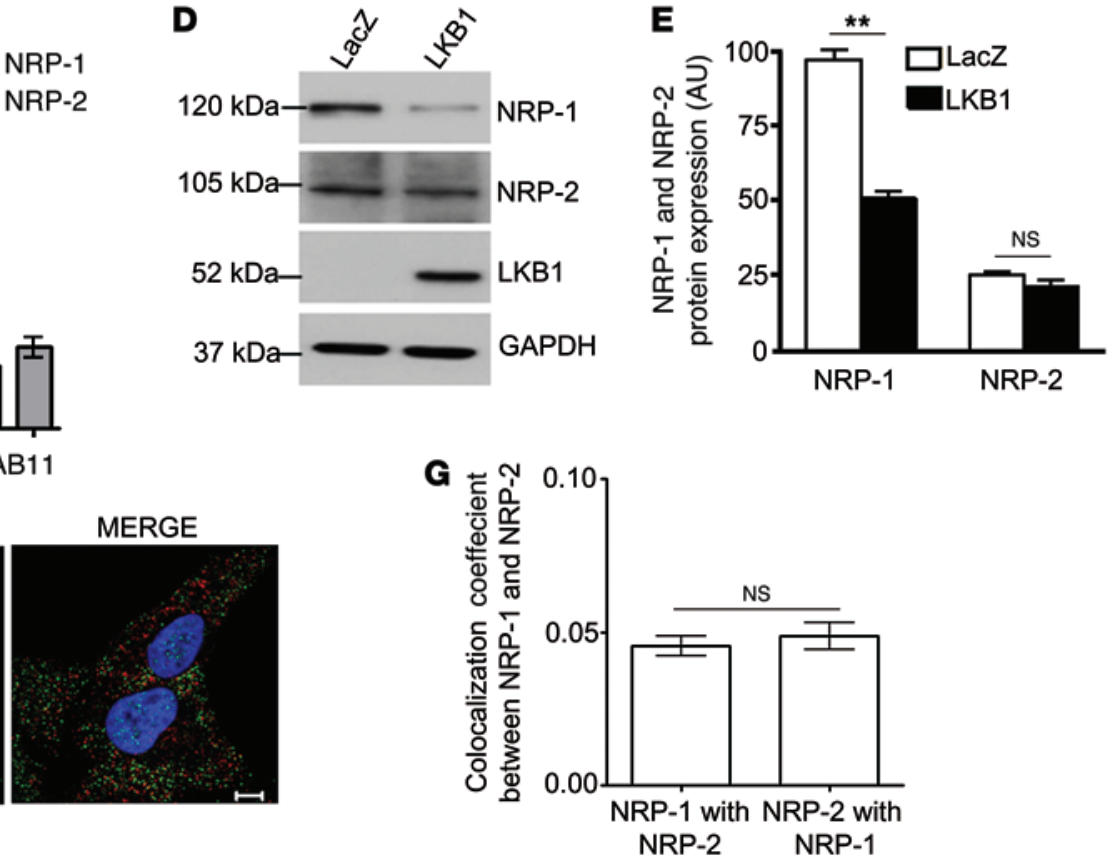

Figure 5. NRP-1 localizes within RAB7 endosomes. (A) Colocalization analysis of NRP-1 (green) with canonical endocytic markers, namely, RAB5 (early), RAB7 (late), and RAB11 (recycling) endosomes (red), using confocal microscopy. DAPI staining (blue). Scale bars: $5 \mu \mathrm{m}$. (B) Similar colocalization analysis was performed with NRP-2 isoform (green) and the RABs (red). Scale bar: $5 \mu \mathrm{m}$. (C) Colocalization coefficients of NRP-1 or NRP-2 with the RABs. Mean \pm SD is shown $(n>50)$. (D and E) NRP-1 or NRP-2 expression was detected by immunoblot analysis in LacZ- or LKB1-transfected A549 cells; mean \pm SD is shown $(n=3)$. ( $F$ and $\mathbf{G}$ ) Colocalization analysis between NRP-1 (red) and NRP-2 (green). DAPI staining (blue). Mean \pm SD is shown $(n>50)$. Scale bars: $5 \mu \mathrm{m} .{ }^{* *} P<0.01,{ }^{* * *} P<0.001$.

tumor growth was observed in all LKB1-NRP-1- cell recipients, the tumors were smaller in size $(17.12 \pm 1.43 \mathrm{mg})$ compared with LKB1 ${ }^{-N R P}-1^{+}$cell recipients (Figure 7, D and E). Tumor development in $\mathrm{LKB1}^{-} \mathrm{NRP}-1^{-}$recipient mice is suggestive of contributions from other gene mutations or aberrant processes present in cancer cells. Despite differences in tumor development, there was no significant variability in organ weight between mouse groups (Supplemental Figure 13). IHC of recovered tumors revealed strong NRP-1, VEGFR2, PDGFR, and FGFR expression in tumors of the $\mathrm{LKB1}^{-} \mathrm{NRP}-1^{+}$group relative to those of all other groups (Figure $7 F$ ). Overall, our results indicate that
LKB1-mediated attenuation of NRP-1 expression correlates with decreased tumor growth and angiogenesis in vivo.

\section{Discussion}

Receptor internalization and subsequent trafficking to the lysosome promotes protein degradation and is a key biological process in terminating signal transduction. Here, we report a novel trafficking function for LKB1 that results in specific NRP-1 abrogation. We observed that hypoxia promoted LKB1 nuclear export to the cytosol, where it interacted with NRP-1 and RAB7. Mechanistically, we found that LKB1 accentuated complex 


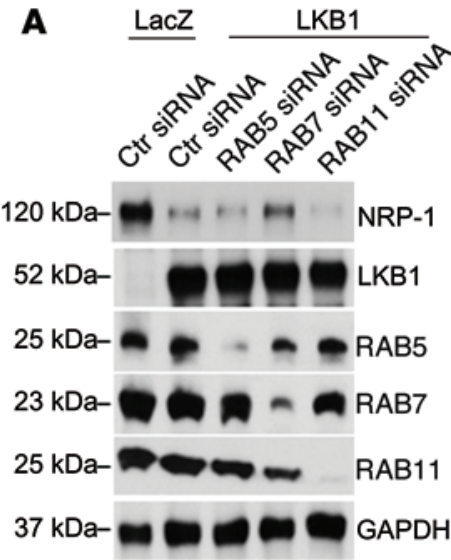

B

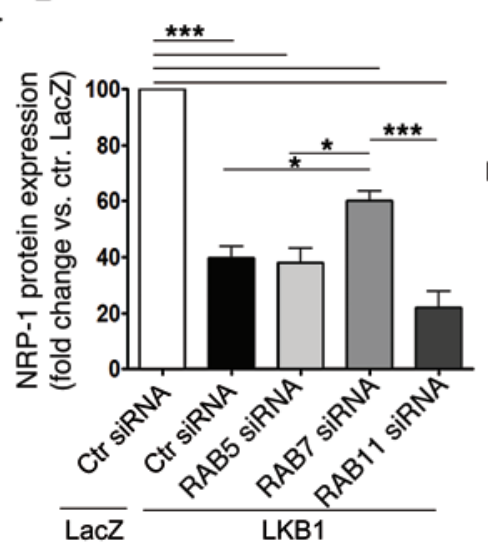

E

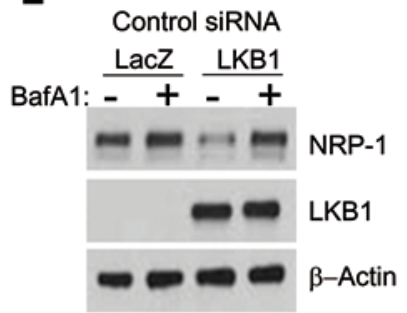

C
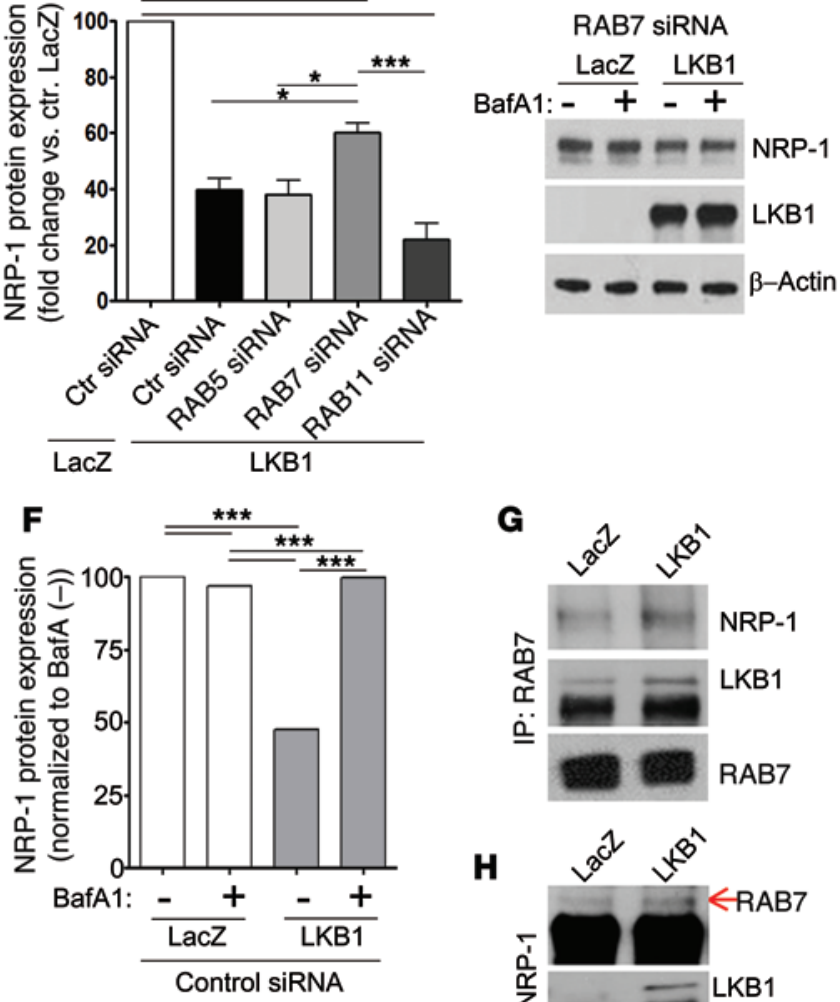

$\mathbf{K}$

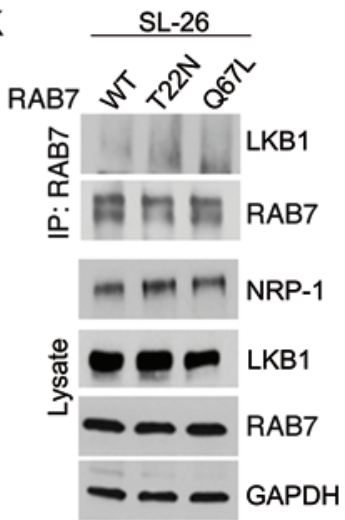

G

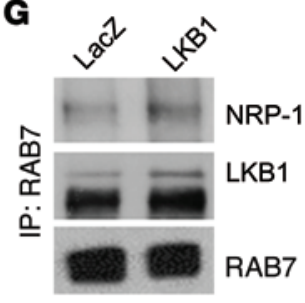

H

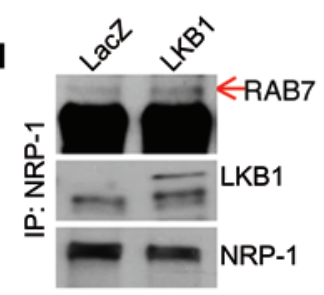

L
D
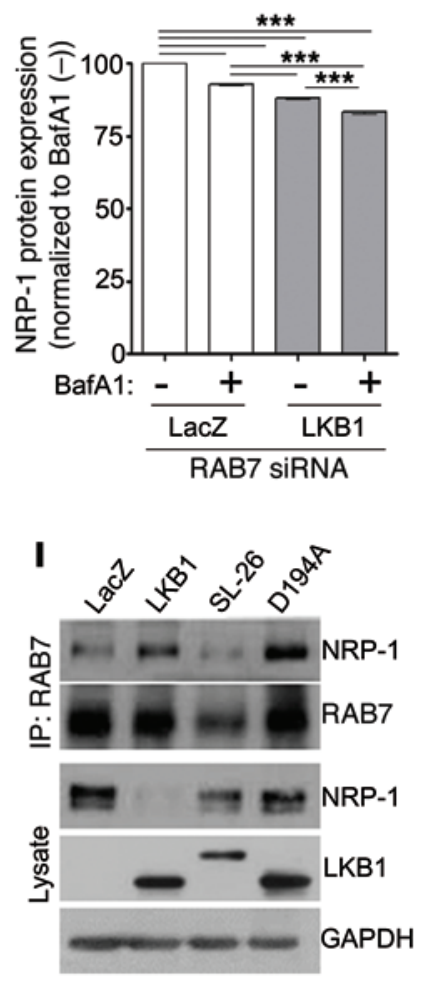

$\mathbf{J}$

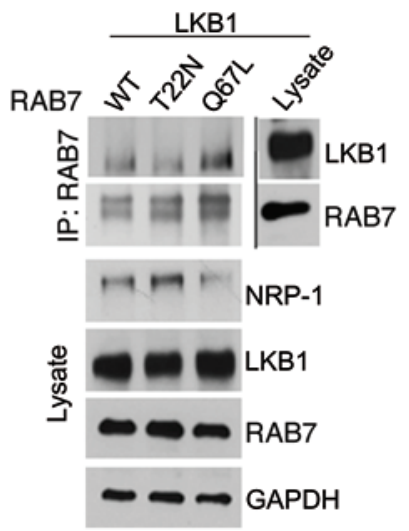

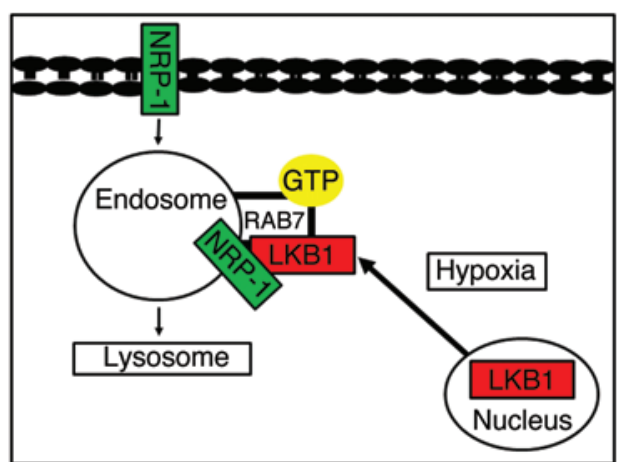

Figure 6. LKB1 is a novel RAB7 effector protein. (A and B) Knockdown (siRNA) of RAB5, RAB7, or RAB11 expression in H1792 cells and detection by immunoblot analysis of NRP-1 and LKB1 protein levels. Mean \pm SD is shown $(n=3)$. (C and D) RAB7 depletion (siRNA) in LacZ- or LKB1-transfected A549 cells with or without lysosome inhibition using BafA1, and detection of NRP-1 and LKB1 expression. Mean \pm SD is shown $(n=3)$. ( $(\mathbf{E}$ and $\mathbf{F})$ Control siRNA in LacZ- or LKB1-transfected A549 cells with or without BafA1-mediated lysosome inhibition, and detection of NRP-1 and LKB1 expression. Mean \pm SD is shown ( $n=3$ ). (C) RAB7 pulldown in A549 cells transfected with LacZ or LKB1 and blotted with NRP-1, RAB7, or LKB1 antibodies. (H) Reverse pulldown of NRP-1 and blotting with RAB7, NRP-1, or LKB1 antibodies. (I) RAB7 pulldown in A549 cells transfected with wild-type LKB1, D194A, or SL-26 and blotted with RAB7 or NRP-1 antibodies. (J and K) GTP-bound RAB7 (Q67L) or GDP-bound RAB7 (T22N) constructs were coexpressed with wild-type LKB1 or SL-26 in A549 cells, prior to RAB7 pulldown and immunoblotting with RAB7, NRP-1, or LKB1 antibodies. (L) Model for LKB1-instigated attenuation of NRP-1 expression. ${ }^{*} P<0.05$, ${ }^{* * *} P<0.001$.

formation of NRP-1 and RAB7 in late endosomes. LKB1 specifically bound the GTP-bound Q67L RAB7 construct, but not the dominant-negative GDP-bound $\mathrm{T} 22 \mathrm{~N}$ construct, promoting rapid transfer and lysosome degradation of NRP-1. Finally, we found that LKB1-instigated loss of NRP-1 expression correlated with decreased tumor angiogenesis and growth in vivo. Overall, our results demonstrated for the first time that LKB1 functions as a RAB7 effector and suppresses angiogenesis by promoting cellular trafficking of NRP-1 from RAB7 vesicles to the lysosome for degradation. 


\section{A}

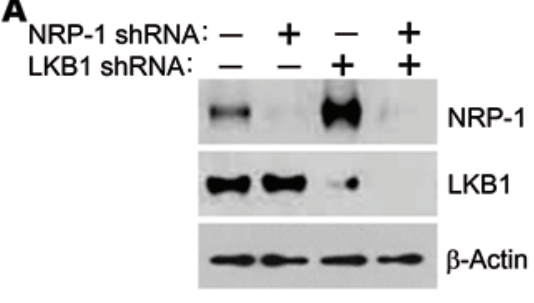

C

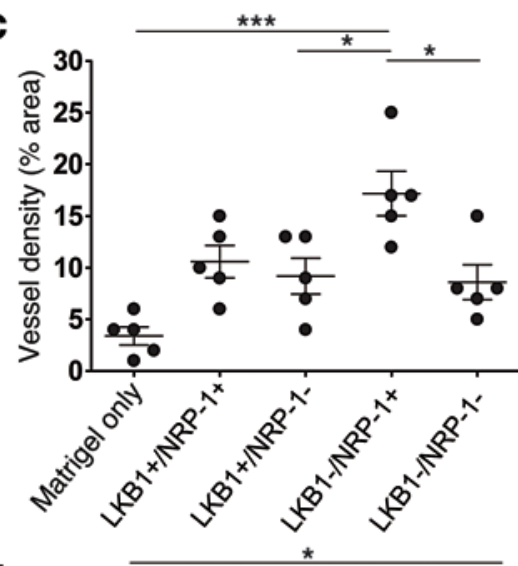

E

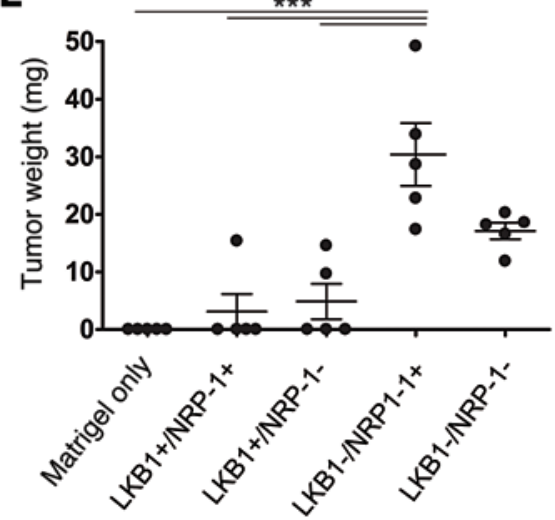

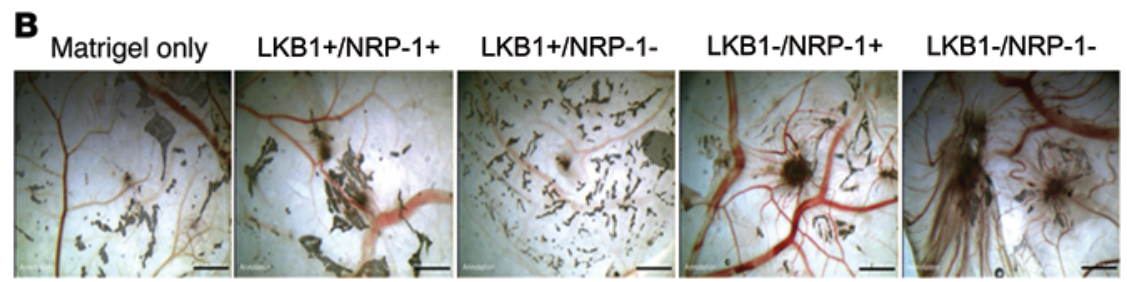

D
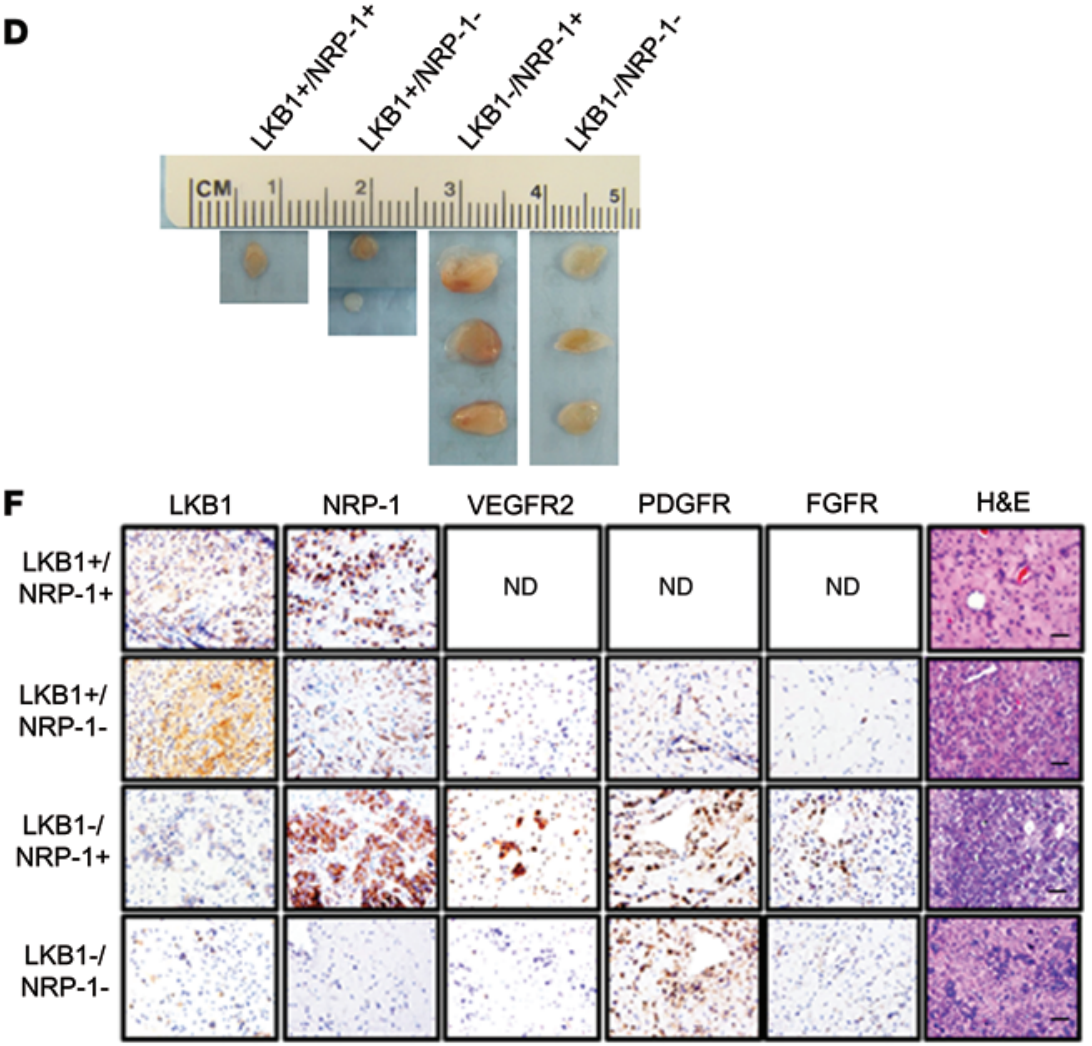

Figure 7. LKB1-mediated NRP-1 inhibition correlates with decreased tumor angiogenesis and growth in vivo. (A) Stable depletion of LKB1 and/or NRP-1 expression was confirmed in $\mathrm{H} 1792$ cells by Western blot analysis. (B and $\mathbf{C}$ ) Vessel density after implantation of H1792 cells with the indicated depletion of LKB1 and/or NRP-1 expression. Scale bars: $0.32 \mathrm{~mm}$. Mean \pm SD is shown ( $n=5 ; 2$ independent repeats). (D and E) H1792 cells with the indicated depletion of LKB1 and/or NRP-1 expression were injected subcutaneously into nude mice, and tumor growth was monitored for 30 days. Representative image of tumors extracted from nude mice. Mean \pm SEM is shown ( $n=5 ; 2$ independent repeats). (F) Paraffin sections of extracted tumors from the different recipient groups were stained for angiogenic receptors, NRP-1, VEGFR2, PDGFR, FGFR, and H\&E (original magnification, $\times 40$ ). ND, not determined due to insufficient tumor sample. Scale bars: $50 \mu \mathrm{m} .{ }^{*} P<0.05,{ }^{* * *} P<0.001$.

Our mechanistic insight into NRP-1 degradation by LKB1 prompted focus on the endocytic pathway. We found specific colocalization of NRP-1 with RAB7 endosomes; however, LKB1 expression resulted in loss of NRP-1 punctate staining. This suggested that LKB1 may play a role in facilitating delivery of the RAB7 cargo (NRP-1) to the lysosome for degradation. Depletion of LKB1 or RAB7 proteins resulted in strong recovery of NRP-1 expression, emphasizing the importance of both proteins in coordinated targeting of the receptor. RAB7 is intimately linked to transport of cargo to the lysosome, and lysosome inhibition corresponded with recovery of NRP-1 expression. Effector proteins that preferentially bind GTP-bound RAB7 are critical to its function. Several RAB7interacting proteins, including RILP and Rubicon, have been shown to provide negative or positive RAB7 effector function $(33,34)$.
We found that LKB1 efficiently bound to the GTP-bound Q67L RAB7 construct, but only weakly to the dominant-negative GDPbound T22N construct. The preferential interaction of LKB1 with Q67L and subsequent NRP-1 degradation are suggestive of a downstream RAB7 effector function for LKB1. Importantly, the nuclear-localized LKB1 mutant SL-26, which lacks cytosolic distribution, failed to interact with RAB7 constructs. Furthermore, LKB1 shares a number of related properties with the RAB7 protein. First, both proteins possess prenylation or farnesylation posttranslational protein modifications on the $\mathrm{C}$-terminal domain required for membrane fusion $(36,45)$. Second, both have been implicated in actin- and microtubule-based processes (32, 46-48). Finally, LKB1 and RAB7 are involved in regulation of cell signaling, autophagy, growth, survival, and development (35-37, 48). 
The potential involvement of autophagy in NRP-1 degradation, considered in light of the association of RAB7 or LKB1 with the autophagic process, was excluded (Supplemental Figure 14). However, actin-cytoskeleton/membrane disruption resulted in recovery of NRP-1 expression (Supplemental Figure 15), supporting roles for both proteins with the actin cytoskeleton.

In the present study, we demonstrated that LKB1 inhibits tumor growth and angiogenesis via enhanced RAB7-mediated lysosome degradation of NRP-1. There is evidence of a proangiogenic effect for LKB1 under hypoxic conditions $(13,49)$, which may involve AMPK, a well-characterized LKB1 downstream enzyme. Here, attenuated NRP-1 expression by LKB1 was independent of AMPK (data not shown). However, the antiangiogenic effects of LKB1 reported herein are consistent with tumor suppressor functions previously attributed to $\operatorname{LKB} 1(8,10,12)$. Our findings have several implications for cancer patients, given the limited benefits of current VEGF/VEGFR2 therapies (50-53). Negligible VEGFR2 or VEGF expression in the cancer cell lines investigated are indicative of limited contributions of these targets to tumor angiogenesis and growth. We speculate that independent NRP-1 functions are sufficient to promote tumor growth and angiogenesis. Hence, the blockade of aberrant NRP-1 expression may provide better outcomes, and targeting LKB1 fulfills these requirements. Internalized NRP-1 receptors found in GTP-bound RAB7 vesicles are subject to enhanced lysosome degradation by LKB1, which results in decreased tumor growth and angiogenesis. However, the specificity with respect to other membrane proteins requires further elucidation. Further investigations are also required to fully characterize and delineate the functions of LKB1 with the RAB GTPase family.

In summary, we demonstrated for the first time that LKB1 suppresses angiogenesis by promoting the cellular trafficking of NRP-1 from RAB7 vesicles to the lysosome for degradation. Loss of LKB1 function might contribute to lung cancer growth and metastasis by instigating angiogenesis switch.

\section{Methods}

Cell culture. MRC-9 cells and all lung cancer cell lines were purchased from American Type Culture Collection and maintained at $37^{\circ} \mathrm{C}$ in a humidified atmosphere of $5 \% \mathrm{CO}_{2}$ in an open-air incubator with full medium consisting of DMEM or RPMI-1640 medium (Lonza or Mediatech) supplemented with $10 \%(\mathrm{v} / \mathrm{v})$ heat-inactivated FBS, $2 \mathrm{mM}$ glutamine, $100 \mathrm{U} / \mathrm{ml}$ penicillin, and $0.1 \mathrm{mg} / \mathrm{ml}$ streptomycin.

LKB1 mutants and plasmid constructs. The LKB1 mutants D194A and S428A were generated as previously described $(41,42)$. D194A is a kinase-inactive LKB1 mutant, whereas S428A cannot be phosphorylated at the canonical serine 428 residue. Plasmids encoding EGFP-fused SL-26 was a gift from L. Dong (University of Texas Health Sciences Center, San Antonio, Texas, USA). Specifically, SL-26 is a constitutively nuclear localized LKB1 mutant originally identified in PJS patients. The mutation is due to deletion of LKB1 amino acids 303-306. Wild-type, T22N (GDP-bound), and Q67L (GTP-bound) $\mathrm{RAB7}$ constructs were generated in our laboratory.

Transient transfections and siRNA. Lung cancer cell lines were routinely passaged 24 hours before transfection and transfected at approximately $70 \%$ confluency. Cells were transfected overnight in 1\% FBS media using the Lipofectamine 2000 protocol (Invitrogen) before incubation under hypoxic conditions or further manipulation.
RNA interference. Stable LKB1- and/or NRP-1-deficient H1792 cell line was achieved using lentiviral shRNAs in pLKO.1-puro plasmid (Santa Cruz Biotechnology) under puromycin selection.

Reagents and cell treatments. Z-Leu-Leu-Leu-aldehyde, Z-LLLCHO (MG-132, $1 \mu \mathrm{M}$; Sigma-Aldrich) or lactacystin (20 $\mu \mathrm{M}$, Enzo Life Sciences) was used for proteosome inhibition; chloroquine $(100 \mu \mathrm{M}$, Invitrogen) or bafilomycin $\mathrm{A}(0.1 \mu \mathrm{M}$, Calbiochem) was used to inhibit the lysosome. All treatments were at 3 hours. For VEGF stimulation, serum-starved cells were stimulated with $25 \mathrm{ng} / \mathrm{ml}$ VEGF (PeproTech) for 5 or 30 minutes. For hypoxia, serum-starved cells were typically maintained under hypoxia for 3 hours, unless otherwise indicated. Hypoxic conditions involved a humidified atmosphere of $5 \% \mathrm{CO}_{2}$ and 1\% oxygen (Hypoxia chamber; BioSpherix) for the indicated times. Control cells were treated with vehicle only (DMSO or PBS) and maintained under normal or hypoxic conditions.

Western blots and immunoprecipitation. These procedures were carried out as previously described $(41,42)$, and blots were probed with specific antibodies according to the manufacturer's suggestions. Primary antibodies were as follows: LKB1 and GAPDH anti-mouse monoclonal antibody (Santa Cruz Biotechnology); anti-rabbit polyclonal RAB5, RAB7, and RAB11 (Cell Signaling); anti-rabbit polyclonal NRP-1, VEGF, Cav-1, and Lamp2 (Abcam); anti-rabbit HIF-1 $\alpha$ (Novus Biologicals). Band intensities were measured and quantified by densitometry (GS-700 Imaging Densitometer; Bio-Rad) after background subtraction from calculated area. Quantification of Western blot was based on the ratio of target protein to GAPDH or $\beta$-actin housekeeping protein and then normalized to the untreated, LacZ, or control siRNA group.

Serum-starved and/or hypoxia-exposed cells were used for immunoprecipitation experiments with respective antibodies.

Cell fractionation. Nuclear/Cytosol Fractionation Kit (BioVision) was used according to the manufacturer's instructions. Quantification of Western blot was based on normalization of LKB1 expression to nuclear (H2AX) or cytosolic (GAPDH) housekeeping proteins in A549 cells transfected with wild-type LKB1 or SL-26 plasmids.

RT-PCR. Primers were used that amplified a 277-bp fragment of LKB1 (forward, 5'-GTTCATCCACCGCATCGAC-3'; reverse, 5'-CACATCCACCAGCTGGATG-3'), a 121-bp fragment of NRP1 (forward, 5'-AAATGCGAATGGCTGATTCAG-3'; reverse, 5'-CTCCATCGAAGACTTCCACGTAGT-3'), a 279-bp fragment of VEGF (forward, 5'-ATCACCATGCAGATTATGCGG-3'; reverse, 5'-CAAATGCTTTCTCCGCTCTGA-3'), and HM18s (forward, 5'-GTCTGTGATGCCCTTAGATG-3'; reverse, 5'-AGCTTATGACCCGCACTTAC-3'). Real-time quantification was performed by SYBR Green (Bio-Rad) with the C1000 thermal cycler, CFX96 detection system (Bio-Rad). Relative gene expression was normalized to HM18s and compared using the $\Delta \Delta \mathrm{Ct}$ method.

Confocal microscopy. Cells were plated on glass coverslips and fixed in $4 \%$ paraformaldehyde. Cell were permeabilized with either $0.1 \%$ Triton $\mathrm{X}-100$ or $0.2 \%$ saponin and blocked with $0.1 \%$ BSA prior to antibody addition. Indirect immunofluorescence experiments were performed using the following primary antibodies: anti-mouse LKB1 (1:75; Santa Cruz), anti-goat NRP-1 (1:100; Santa Cruz), anti-rabbit NRP-1 (1:100; Santa Cruz), anti-goat NRP-2 (1:100; Santa Cruz), anti-rabbit RAB5, RAB7, and RAB11 (1:100; Cell Signaling), anti-rabbit Lamp2 (1:750; Abcam). Secondary antibodies consisted of Alexa Fluor 488, 555, and 647 (donkey anti-goat, -rabbit, or -mouse, respectively) used at 1:400 dilution. For LC3-GFP expression, the viral vector was added to the 
media of cells after 8 hours of transfection and incubated for an additional 16 hours. For rhodamine-labeled transferrin, cells were incubated on ice for 10 minutes with rhodamine in the dark, followed by incubation at $37^{\circ} \mathrm{C}$ for 5 minutes or 30 minutes prior to fixation. Imaging was carried out using a Zeiss LSM 510 or 710 confocal microscope with a $\times 63$ objective. Quantitative nuclear to cytoplasmic distribution was done using LSM image software. Briefly, regions of interest identifying the nucleus (DAPI) and cytoplasm were determined, and fluorescence intensity was measured. The colocalization coefficient was quantified using Zen 2011 software, which corresponds to the Zeiss 710 microscope. For each channel, the colocalization coefficient was calculated as the ratio of pixels exhibiting colocalized NRP-1 to total pixels exhibiting NRP-1 staining (54). All quantitation was done using original, unmodified data images. Contrast and brightness were altered equally for all images to enhance publication quality. Treatment conditions had the following durations: VEGF, 30 minutes; hypoxia, 3-4 hours; transferrin, 5 or 30 minutes.

CAM assay. CAM assay was modified from a previously reported procedure (55). Fertilized specific pathogen-free chicken eggs were obtained from Charles River Laboratories at incubation day 0. Eggs were incubated at $100^{\circ} \mathrm{F}$ and $60 \%-65 \%$ humidity for 10 days (incubator model 1502; GQF Manufacturing Co.). Cells in Matrigel (1:1) were implanted subcutaneously into the CAMs of 10-day-old chick embryos through a hole made in the superior surface of the eggshell. The shell windows were protected with paper tape and subsequently incubated for an additional 72 hours. Matrigel with serum-free media was used as the negative control. CAMs were harvested, fixed with $4 \%$ paraformaldehyde in $0.1 \%$ Triton $\mathrm{X}-100$, and imaged digitally (Xli usb 2.0 digital camera) using a stereoscope with $\times 0.3$ auxiliary objective and Xli cap software. 2 independent CAM assays were performed using 3-6 eggs per group. Angiogenesis was assessed via image analysis by analyzing the convergence of blood vessels toward the implant. The total number of blood vessel branch points converging toward the tumor implant was scored by observers blinded to experimental conditions.

Nude mice experiment. Approximately $1 \times 10^{6} \mathrm{H} 1792$ cells with intact or depleted LKB1 and/or NRP-1 expression were injected subcutaneously into 8-week-old nude mice (Charles River). Mice were monitored daily, and tumors were extracted after 30 days.

IHC. Tissues were fixed in $10 \%$ ice-cold formalin overnight, then embedded in paraffin. Briefly, slides were deparaffinized and dehy- drated for 30 minutes at room temperature. Sections were incubated for 30 minutes in diluted normal blocking serum, and primary antibodies were added to slides after excess serum had been removed. Respective antibodies against LKB1, NRP-1, VEGFR2, VEGF, PDGFR, and FGFR (Lifespan Biosciences) were diluted according to the manufacturer's instructions. Analyzed data were scored by blinded analyses of expression levels (1, low; 2, moderate; 3, high). For H\&E staining, tissues were collected, fixed in $10 \%$ ice-cold formalin overnight, and then embedded in paraffin. Serial paraffin sections $(4 \mu \mathrm{m})$ were obtained and then treated with H\&E staining.

Statistics. Data are expressed as mean \pm SEM or mean \pm SD as indicated in the figure legends. Statistical comparisons were performed using unpaired 2-tailed Student's $t$ test or 1-way ANOVA with Bonferroni multiple-comparison test. A $P$ value less than 0.05 was considered significant.

Study approval. Informed subject consent was obtained, and ethical approval to carry out the expression analysis on snap-frozen samples from lung cancer patients was approved by Qilu Hospital of Shandong University Research Ethical Committee. The experiment involving nude mice was approved by OUHSC IACUC (protocol no. 11-070-H).

\section{Acknowledgments}

This work was supported by NIH grants AG047776, HL079584, HL080499, HL074399, HL089920, HL096032, HL105157, and HL110488 to M-.H. Zou. We thank Najeeb Shirwany for helpful discussions, Chaoyong He for technical assistance, R. Guo for assistance with pathological characterization of tumors extracted from nude mice, Zitong Wang for technical assistance and data entry, and the Oklahoma Medical Research Foundation (OMRF) imaging facility for assistance with confocal microscopy.

Address correspondence to Ming-Hui Zou, BSEB 306A, Department of Medicine, University of Oklahoma Health Sciences Center, 941 Stanton L. Young Blvd., Oklahoma City, Oklahoma 73104, USA. Phone: 405.271.3974; E-mail: ming-hui-zou@ouhsc.edu.

Cheng Zhang's present address is: Key Laboratory of Cardiovascular Remodeling and Function Research, Chinese Ministry of Education and Chinese Ministry of Public Health, Qilu Hospital of Shandong University, Jinan, Shandong, China.
1. Gurumurthy S, et al. The Lkb1 metabolic sensor maintains haematopoetic stem cell survival. Nature. 2010;468(7324):659-663.

2. Shaw RJ, et al. The LKB1 tumor suppressor negatively regulates mTOR signaling. Cancer Cell. 2004;6(1):91-99.

3. Lizcano JM, et al. LKB1 is a master kinase that activates 13 kinases of the AMPK subfamily, including MARK/PAR-1. EMBO J. 2004;23(4):833-843.

4. Brugarolas J, Kaelin JWG. Dysregulation of HIF and VEGF is a unifying feature of the familial hamartoma syndromes. Cancer Cell. 2004;6(1):7-10.

5. Veeranki S, Hwang S, Sun T, Kim B, Kim L. LKB1 regulates development and the stress response in Dictyostelium. Dev Biol. 2011;360(2):351-357.

6. Ylikorkala A, et al. Vascular abnormalities and deregulation of VEGF in Lkb1-deficient mice. Science. 2001;293(5533):1323-1326.

7. Ding L, et al. Somatic mutations affect key pathways in lung adenocarcinoma. Nature. 2008;455(7216):1069-1075.

8. Matsumoto S, et al. Prevalence and specificity of LKB1 genetic alterations in lung cancers. Oncogene. 2007;26(40):5911-5918.

9. Shah U, Sharpless NE, Hayes DN. LKB1 and lung cancer: more than the usual suspects. Cancer Res. 2008;68(10):3562-3565.

10. Zhuang Z, Di G, Shen Z, Ding J, Shao Z. Enhanced expression of LKB1 in breast cancer cells attenuates angiogenesis, invasion, and metastatic potential. Mol Cancer Res. 2006;4(11):843-849.

11. Sanchez-Cespedes M. A role for LKB1 gene in human cancer beyond the Peutz-Jeghers syn- drome. Oncogene. 2007;26(57):7825-7832.

12. Hongbin J, et al. LKB1 modulates lung cancer differentiation and metastasis. Nature. 2007;448(7155):807-811.

13. Ohashi K, Ouchi N, Higuchi A, Shaw RJ, Walsh K. LKB1 deficiency in Tie2-Cre-expressing cells impairs ischemia-induced angiogenesis. J Biol Chem. 2010;285(29):22291-22298.

14. Londesborough A. et al. LKB1 in endothelial cells is required for angiogenesis and TGF $\beta$ mediated vascular smooth muscle cell recruitment. Development. 2008;135(13):2331-2338.

15. Staton CA, Kumar I, Reed MWR, Brown NJ. Neuropilins in physiological and pathological angiogenesis. J Pathol. 2007;212(3):237-248.

16. Bielenberg DR, Pettaway CA, Takashima S, Klaagsbrun M. Neuropilins in neoplasms: expression, regulation, and function. Exp Cell Res. 
2006;312(5):584-593.

17. Jubb AM, et al. Neuropilin-1 expression in cancer and development. J Pathol. 2011;226(1):50-60.

18. Ellis LM. The role of neuropilins in cancer. Mol Cancer Ther. 2006;5(5):1099-1107.

19. Pellet-Many C, Frankel P, Jia H, Zachary I. Neuropilins: structure, function and role in disease. Biochem J. 2008;411(2):211-226.

20. Miao HQ, Lee P, Lin H, Soker S, Klagsbrun M. Neuropilin-1 expression by tumor cells promotes tumor angiogenesis and progression. FASEB J. 2000;14(15):2532-2539.

21. Oh H, et al. Selective induction of neuropilin-1 by vascular endothelial growth factor (VEGF): a mechanism contributing to VEGFinduced angiogenesis. Proc Natl Acad Sci US A. 2002;99(1):383-388.

22. Jia H, et al. Neuropilin-1 antagonism in human carcinoma cells inhibits migration and enhances chemosensitivity. Br J Cancer. 2010; 102(3):541-552.

23. Rizzolio S, et al. Neuropilin-1-dependent regulation of EGF-receptor signaling. Cancer Res. 2012;72(22):5801-5811.

24. Zachary IC. How neuropilin-1 regulates receptor tyrosine kinase signaling: the knowns and known unknowns. Biochem Soc Trans. 2011;39(6):1583-1591.

25. Wang L, Mukhopadhyay D, Xu X. C terminus of RGS-GAIP-interacting protein conveys neuropilin-1-mediated signaling during angiogenesis. FASEB J. 2006;20(9):1513-1515.

26. Soker S, Takashima S, Miao HQ, Neufeld G, Klagsbrun M. Neuropilin-1 is expressed by endothelial and tumor cells as an isoform-specific receptor for vascular endothelial growth factor. Cell. 1998;92(6):735-745.

27. Lee $\mathrm{P}$, et al. Neuropilin-1 is required for vascular development and is a mediator of VEGFdependent angiogenesis in zebrafish. Proc Natl Acad Sci U S A. 2002;99(16):10470-10475.

28. Douglas H, Folkman J. Patterns and emerging mechanisms of the angiogenic switch during tumorigenesis. Cell. 1996;86(3):353-364.

29. Bergers G, Benjamin LE. Tumorigenesis and the angiogenic switch. Nat Rev Cancer. 2003;3(6):401-410.

30. Ballmer-Hofer K, Andersson AE, Ratcliffe LE, Berger P. Neuropilin-1 promotes VEGFR2 traf- ficking through Rab11 vesicles thereby specifying signal output. Blood. 2011;118(3):816-826.

31. Evans IM, et al. Neuropilin-1 signaling through p130Cas tyrosine phosphorylation is essential for growth factor-dependent migration of glioma and endothelial cells. Mol Cell Biol. 2011;31(6):1174-1185.

32. Zhang M, Chen L, Wang S, Wang T. Rab7: roles in membrane trafficking and disease. Biosci Rep. 2009;29(3):193-209.

33. Cantalupo G, Alifano P, Robert V, Bruni CB, Bucci C. Rab-interacting lysosome protein (RILP): the Rab7 effector required for transport to lysosomes. EMBO J. 2001;20(4):683-693.

34. Sun Q, Westphal W, Wong KN, Tan I, Zhong Q. Rubicon controls endosome maturation as a Rab7 effector. Proc Natl Acad Sci U S A. 2010;107(45):19338-19343.

35. Vanlandingham PA, Ceresa BP. Rab7 regulates late endocytic trafficking downstream of multivesicular body biogenesis and cargo sequestration. J Biol Chem. 2009;284(18):12110-12124.

36. Pfeffer S, Aivazian D. Targeting Rab GTPases to distinct membrane compartments. Nat Rev Mol Cell Biol. 2004;5(11):886-896.

37. Zerial M, McBride H. Rab proteins as membrane organizers. Nat Rev Mol Cell Biol. 2001;2(2):107-118.

38. Wencheng Z, et al. Endothelial cell-specific liver kinase B1 deletion causes endothelial dysfunction and hypertension in mice in vivo. Circulation. 2014;129(13):1428-1439.

39. McMahon G. VEGF receptor signaling in tumor angiogenesis. Oncologist. 2000;5(suppl 1):3-10.

40. Bae D, Lu S, Taglienti CA, Mercurio A. M. Metabolic stress induces the lysosomal degradation of neuropilin-1 but not neuropilin-2. J Biol Chem. 2008;283(42):28074-28080.

41. Song P, et al. Protein kinase Czeta-dependent LKB1 serine 428 phosphorylation increases LKB1 nucleus export and apoptosis in endothelial cells. J Biol Chem. 2008;283(18):12446-12455.

42. Xie Y, et al. Identification of the serine 307 of LKB1 as a novel phosphorylation site essential for its nucleocytoplasmic transport and endothelial cell angiogenesis. Mol Cell Biol. 2009;29(13):3582-3596.

43. Boudeau J, et al. MO25 $\alpha / \beta$ interact with STRAD $\alpha / \beta$ enhancing their ability to bind, acti- vate and localize LKB1 in the cytoplasm. EMBO J. 2003;22(19):5102-5114

44. Boudeau J, et al. Analysis of the LKB1-STRADMO25 complex. J Cell Sci. 2004; 117(pt 26):6365-6375.

45. Alessi DR, Sakamoto K, Bayascas JR. LKB1dependent signaling pathways. Annu Rev Biochem. 2006;75:137-163.

46. Xu X, Omelchenko T, Hall A. LKB1 tumor suppressor protein regulates actin filament assembly through Rho and its exchange factor $\mathrm{Dbl}$ independently of kinase activity. BMC Cell Biol. 2010;11:77.

47. Zhang S, et al. The tumor suppressor LKB1 regulates lung cancer cell polarity by mediating cdc 42 recruitment and activity. Cancer Res. 2008;68(3):740-748.

48. Partanen JI, Nieminen AI, Mäkelä TP, Klefstrom J. Suppression of oncogenic properties of c-Myc by LKB1-controlled epithelial organization. Proc Natl Acad Sci U S A. 2007;104(37):14694-14699.

49. Nagata D, Mogi M, Walsh K. AMP-activated protein kinase (AMPK) signaling in endothelial cells is essential for angiogenesis in response to hypoxic stress. J Biol Chem. 2003;278(33):31000-31006.

50. Basel S, Janice NA, Harold DF. Anti-VEGF/ VEGFR therapy for cancer: reassessing the target. Cancer Res. 2012;72(8):1909-1914.

51. Geretti E, Klagsbrun M. Neuropilins: novel targets for anti-angiogenesis therapies. Cell Adh Migr. 2007;1(2):56-61.

52. Pan Q, et al. Blocking neuropilin-1 function has an additive effect with anti-VEGF to inhibit tumor growth. Cancer Cell. 2007;11(1):53-67.

53. Hong T, Chen Y, Wu Y. Targeting neuropilin 1 as an antitumor strategy in lung cancer. Clin Cancer Res. 2007;13(16):4759-4768

54. Zinchuk V, Zinchuk O, Okada T. Quantitative colocalization analysis of multicolor confocal immunofluorescence microscopy images: pushing pixels to explore biological phenomena. Acta Histochem Cytochem. 2007;40(4):101-111.

55. Quagliata S, Pacini S, Punzi T, Malentacchi C, Ruggiero M, Delfino G. Bombesin promotes vasculogenesis and angiogenesis in chick chorio-allantoic membrane: A morphometric, structural, and ultrastructural study. J Morphol. 2008;269(1):72-83. 